\title{
Article
}

\section{[2+2+2]-Cycloaddition reactions using immobilized alkynes. A proof of concept for an integral use of the outcoming products in solid-phase synthetic methodologies}

Martin Jorge Riveira, Cintia M. Diez, Mirta P. Mischne, and Ernesto G. Mata

J. Org. Chem., Just Accepted Manuscript • DOI: 10.1021/acs.joc.8b01378 • Publication Date (Web): 29 Jun 2018

Downloaded from http://pubs.acs.org on June 30, 2018

\section{Just Accepted}

"Just Accepted" manuscripts have been peer-reviewed and accepted for publication. They are posted online prior to technical editing, formatting for publication and author proofing. The American Chemical Society provides "Just Accepted" as a service to the research community to expedite the dissemination of scientific material as soon as possible after acceptance. "Just Accepted" manuscripts appear in full in PDF format accompanied by an HTML abstract. "Just Accepted" manuscripts have been fully peer reviewed, but should not be considered the official version of record. They are citable by the Digital Object Identifier (DOI®). "Just Accepted" is an optional service offered to authors. Therefore, the "Just Accepted" Web site may not include all articles that will be published in the journal. After a manuscript is technically edited and formatted, it will be removed from the "Just Accepted" Web site and published as an ASAP article. Note that technical editing may introduce minor changes to the manuscript text and/or graphics which could affect content, and all legal disclaimers and ethical guidelines that apply to the journal pertain. ACS cannot be held responsible for errors or consequences arising from the use of information contained in these "Just Accepted" manuscripts. 
$[2+2+2]-C y c l o a d d i t i o n$ reactions using immobilized alkynes. A proof of concept for an integral use of the outcoming products in solid-phase synthetic methodologies

Martín J. Riveira ${ }^{\dagger}$, Cintia M. Diez ${ }^{\dagger}$, Mirta P. Mischne ${ }^{*}$ and Ernesto G. Mata*

Instituto de Química Rosario, Facultad de Ciencias Bioquímicas y Farmacéuticas, Universidad Nacional de Rosario-CONICET, Suipacha 531, S2002LRK, Rosario, Argentina.

mischne@iquir-conicet.gov.ar, mata@iquir-conicet.gov.ar

\title{
TABLE OF CONTENTS/ABSTRACT GRAPHIC
}

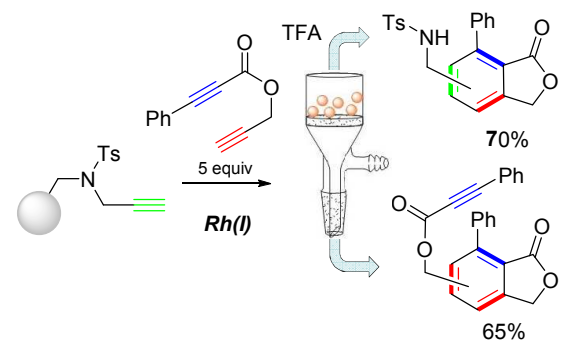

\begin{abstract}
The transition-metal-catalyzed [2+2+2]-cycloaddition of alkynes has become a powerful atom-economical strategy for aromatic ring construction. Unfortunately, the control of the stereo-, regio- and chemoselectivity of these processes is usually challenging and these reactions can potentially lead to complex unuseful mixtures. While solid-phase chemistry has proven to be a successful tool for decreasing the number of cycloadducts formed and for facilitating the purification step, an integral use of the outcoming products in this complex reaction is described herein. By using an immobilized monoalkyne, the transition-metal-catalyzed $[2+2+2]$-cycloaddition with soluble 1,6 -diyne-esters led to the
\end{abstract}


simultaneous preparation of soluble and solid-supported phthalides, showing a new way to benefit from solid-phase synthetic methodologies.

\section{INTRODUCTION}

The preparation of highly-substituted aromatic systems has received considerable attention in organic synthesis as these structural motifs dress-up countless essential pharmaceutical ingredients, natural products and important materials. The construction of accurately decorated molecules of this type has mostly relied on performing substitution and coupling reactions on simpler, more rudimentary aromatic precursors. In the last decades, however, great interest has been devoted to the transition-metal-mediated construction of aromatic rings and, in particular, on the $[2+2+2]$-cycloaddition. ${ }^{1}$ This attractive transition-metal catalyzed transformation cleanly merges three alkyne moieties into an aromatic ring. Depending on the combinations of triple bonds, mono, bi, tri and tetracyclic benzene ring-containing scaffolds can be obtained through inter or intramolecular cycloadditions (Scheme 1).

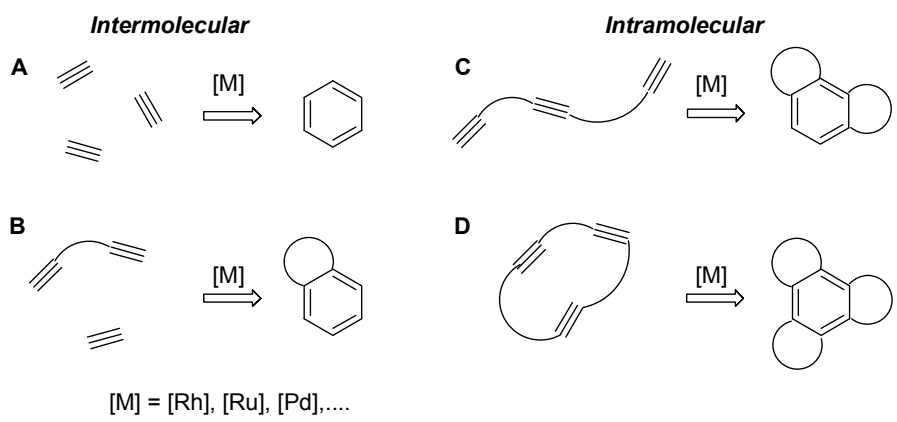

Scheme 1. $[2+2+2]$-Cycloaddition of alkynes

The continuous development of the field led to the successful application of the process to the total synthesis of natural products, ${ }^{2}$ macrocycle construction, ${ }^{3}$ the preparation of molecules for materials science such as dendrimers or polyaromatic hydrocarbons, ${ }^{4}$ and 
the asymmetric synthesis of axially chiral compounds, ${ }^{5}$ just to name a few. ${ }^{6}$ Notwithstanding these advances, it is unarguable that the control of the chemo- and regioselectivity of these processes is a critical issue. Even if side reactions are excluded (e.g. linear di- and trimerizations), ${ }^{7} 38$ possible products can be generated from the combination of three different non-symmetrical alkynes! In this context, several strategies have been developed to control and ultimately simplify the outcome of these apparently unwieldy cycloadditions. Within this context, the group of Takeuchi reported highly selective and controllable Ir-catalyzed [2+2+2]-cycloadditions in which changing the phosphine ligand completely switches the product outcome (Scheme 2A, 2:1 vs. 1:2). ${ }^{8}$ On the other hand, Yamamoto's group reported the formal cyclotrimerization between alkynylboronates, propargyl alcohols and terminal alkynes in which the boron group acts as a tether that controls initial boraruthenacycle construction eventually leading to a single boraphthalide regioisomer that, in turn, can be transformed into diverse attractive aromatic products (Scheme 2B). ${ }^{9}$ In a related approach, silicon-based tethered-[2+2+2]cycloadditions have been reported as well. For improving chemo- and regioselectivity, silyl ethers temporary connect isolated alkyne units in order to transform an intermolecular reaction into an intramolecular one. ${ }^{10}$

The use of immobilized substrates in transition-metal catalyzed processes has been beneficial to overcome selectivity problems. $^{11}$ In particular, the usefulness of $[2+2+2]-$ cycloadditions on solid-supports has been independently demonstrated by the groups of Sun, ${ }^{12}$ Deiters ${ }^{13}$ and Martinez (Scheme 2C). ${ }^{14}$ By using solid-supported diynes, these authors got rid of side-products such as those arising from di- or trimerizations of the diyne components. As in any typical solid-phase strategy, these reactions were driven to 
completion by using an excess of the soluble component, so any non immobilized sideproducts and unused reagents is removed by a simple filtration during work-up procedures.

Addressing the solid-phase synthesis from a different viewpoint, we envisaged a new approach to improve the outcome of the process. We sought to devise a dual-purpose strategy for fully harnessing the solid-phase methodology. We reasoned that, by using an immobilized alkyne in lieu of a diyne, synthetic and biologically relevant products could be formed both in solution and on resin; a process in which no phase is wasted (Scheme 2D).

Herein we report the application of this conceptually new approach to the generation of isolated, soluble and immobilized, substituted phthalides by means of a “convergent" process integrating solution and solid-phase synthesis.
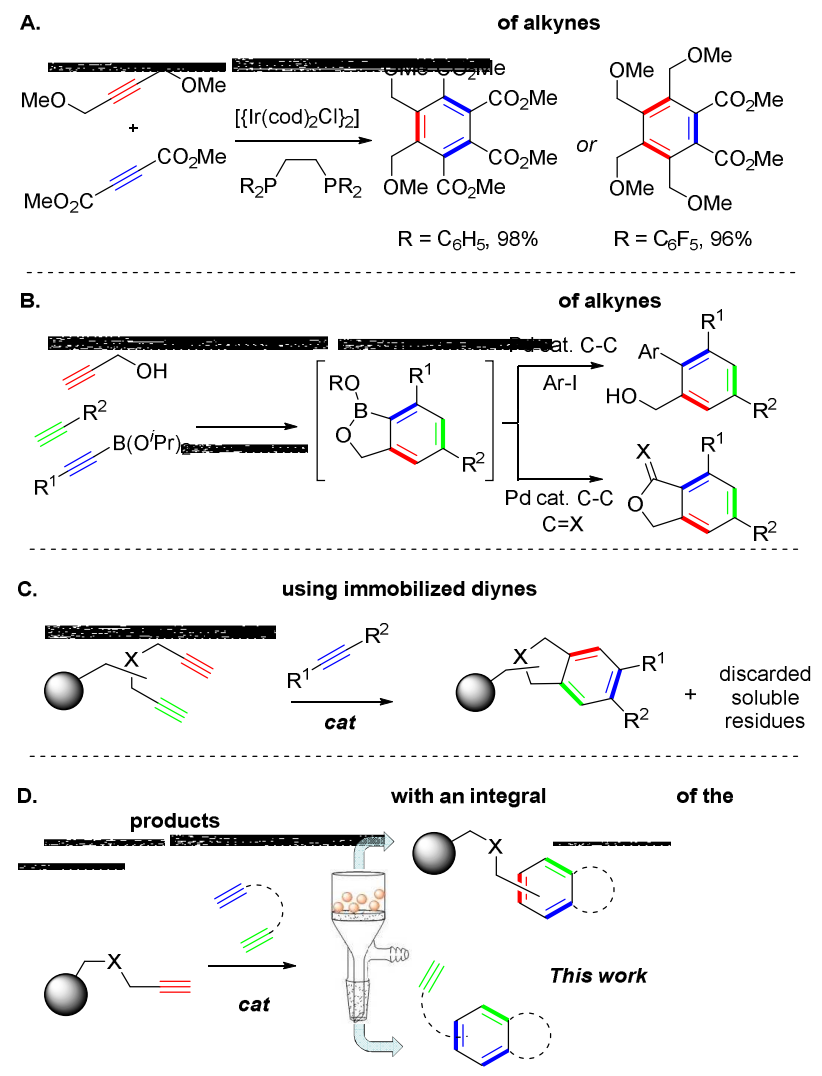

Scheme 2. Facilitated or controlled $[2+2+2]$-cycloadditions of alkynes

\section{RESULTS AND DISCUSSION}


Solution-phase $[2+2+2]-C y c l o a d d i t i o n$ for the Synthesis of Phthalides. In order to establish the basis of the process and to explore the scope of the cycloaddition, our first set of experiments were performed in solution based on a procedure reported by Witulski and Zimmermann which involves the use of Wilkinson's catalyst. ${ }^{15}$ As shown in Table 1, ester-type diynes were used as substrate since the cycloadditions would then lead to phthalide-type products which are attractive structures since these benzolactones are frequently found in naturally occurring substances that exhibit a broad spectrum of biological activities. ${ }^{16-18}$ The other substrate, $N$-benzyl- $N$-propargyl- $p$-toluenesulfonamide (1a), was chosen as the monoyne component for three reasons: the benzyl amine group would be easily translated to solid-phase either through alkylation or substitution strategies, ${ }^{19}$ the behaviour of propargylamines has not been extensively explored in cyclotrimerization reactions and, finally, because there has been recent interest in the biological properties of secondary and tertiary sulfonamides. ${ }^{20}$

Table 1. Solution-phase $[2+2+2]$-cycloaddition for the synthesis of phthalides

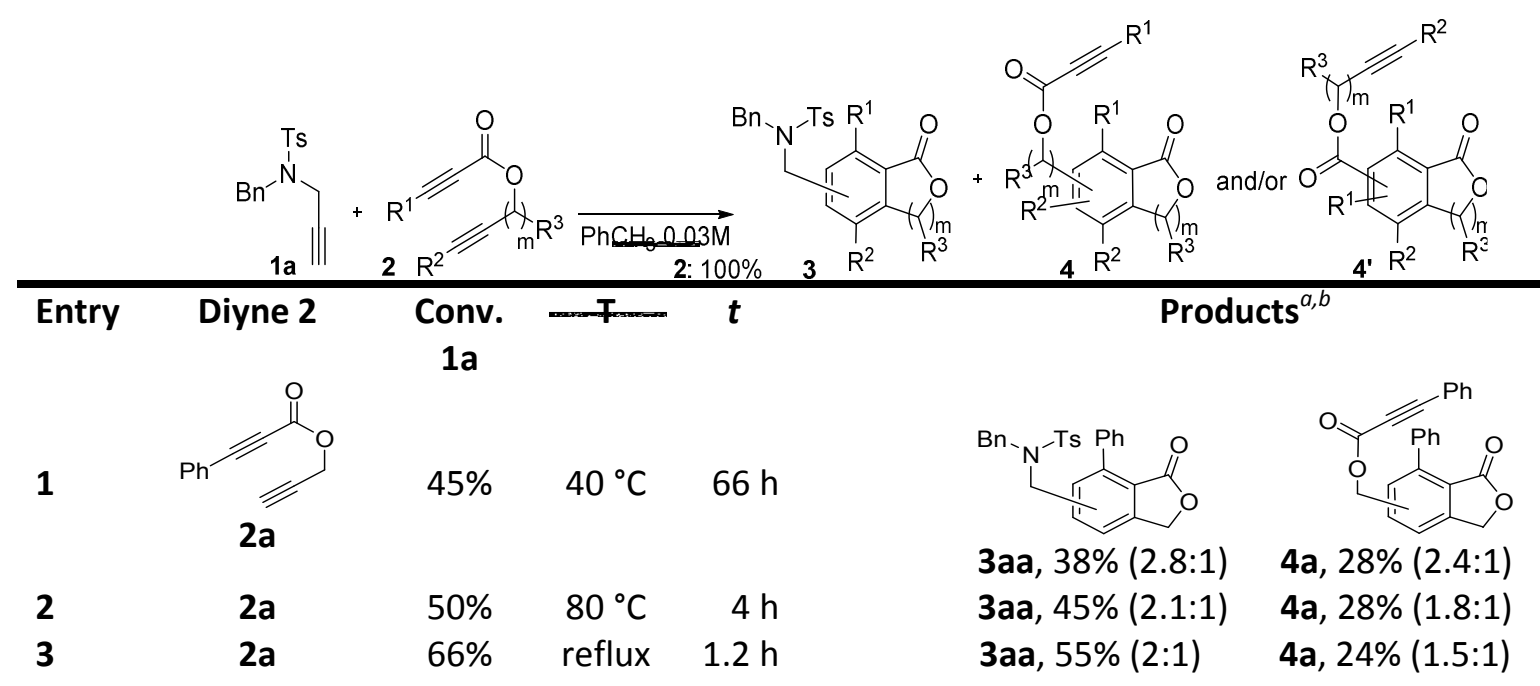


$4 \quad-\quad 25 \%$ reflux $2 \mathrm{~h}$

$5^{c} \quad 2 a \quad<20 \%$ reflux $9 \mathrm{~h}$

$6^{d} \quad 2 a \quad 82 \%$ reflux $1.3 \mathrm{~h}$

72 2a (2 equiv) $74 \%$ reflux $2 \mathrm{~h}$

3aa, traces (3:1)

3aa, 15\% (2.3:1)

3aa, 71\% (2.1:1) 4a, 50\% (1.7:1)

3aa, $76 \%(2.2: 1)$<smiles>O=C1OCc2c1cccc2-c1ccccc1</smiles>

3ab, 46\% (1:1) 4'b, 35\% (2:1)

9

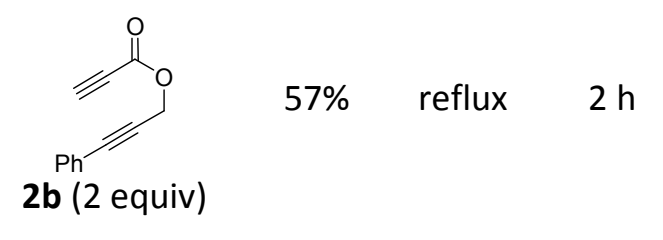

10

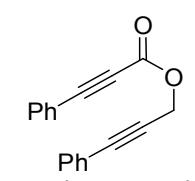

2c (5 equiv)

$99 \%$ reflux

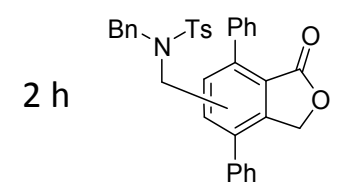

3ac, $64 \%(1.2: 1)$

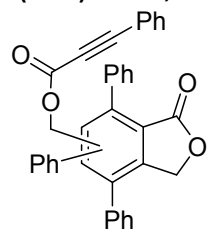

4c, $13 \%(2: 1)$
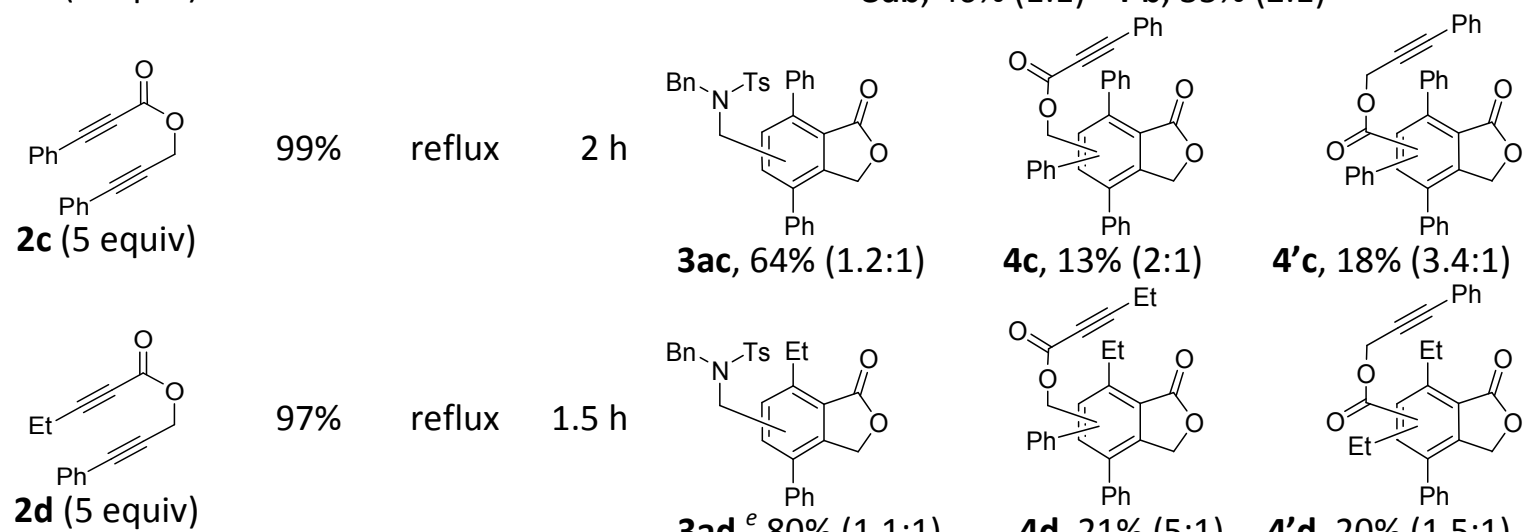

$97 \%$ reflux $\quad 1.5 \mathrm{~h}$

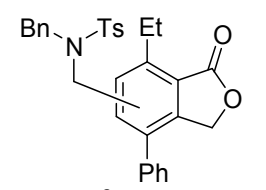

3ad, ${ }^{e} 80 \%$ (1.1:1)

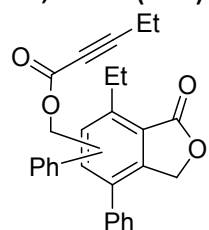

4d, $21 \%(5: 1)$

4'c, $18 \%(3.4: 1)$<smiles>C#CCCOC(=O)C#Cc1ccccc1</smiles>

$55 \%$

reflux

$10 \mathrm{~h}$

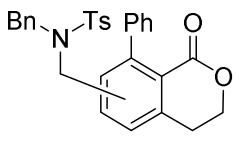

3ae, < 40\% (3:1)

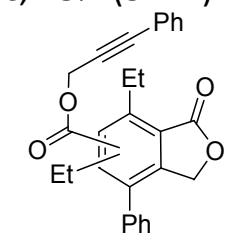

4'd, 20\% (1.5:1)<smiles>C#CCCCOC(=O)C#Cc1ccccc1</smiles>

$86 \%$

reflux

$10 \mathrm{~h}$

As shown in Table 1, the reaction of substrate 1a and a variety of unsymmetrical diynes 2 leads to the formation of cross-cycloaddition products 3 and homodimers 4 . In addition, for many substrates, homotrimers arising from a secondary [2+2+2]-cycloaddition 
event on phthalide products 4 could also be found in the reactions mixtures in yields ranging from 10 to $25 \%$ (see SI). The structures of the cycloadducts were determined based on the analyses of the 1D and 2D NMR data, including in some cases NOE experiments (see SI). Regiochemistry of the obtained compounds was determined by analysis of the ${ }^{1} \mathrm{H}$ NMR spectra of the crude mixtures.

In general, moderate to low regioselectivity was obtained and, although this selectivity was shown to be slightly affected as higher temperatures were reached, this was necessary to guarantee high conversion of substrate 1a (entries 1-3). Indeed, alkyne 1a exhibited poor reactivity, probably due to the bulky tertiary sulfonamide group and, in the absence of any diyne substrate $2, c a .75 \%$ of the starting material could be recovered unaltered after $2 \mathrm{~h}$ under reflux in the presence of Wilkinson cat. (entry 4). Although the production of cross-cycloadducts $\mathbf{3}$, as well as the conversion of substrate $\mathbf{1 a}$, could be raised by using an excess of diyne substrates $\mathbf{2}$ (entries 3 vs 7 and 8), yields of benzolactone-type products $\mathbf{3}$ and $\mathbf{4}$ were in general moderate. It should be noted that the use of other solvents (e.g. EtOH, dichloromethane and chloroform), or other standard Pd and $\mathrm{Ru}$ catalysts that have been previously used for this type of processes, ${ }^{21,22}$ did not provide any improvement (e.g. entries 5-6). While it can instinctively be proposed that this outcome could be due to lack of chemoselectivity, the advantages of a solid-phase approach became apparent from the very beginning. The main disadvantage of this methodology lies in the purification of the crude mixtures thus obtained. For instance, after successive chromatography purifications, benzolactones 3aa could not be efficiently separated from ortho-homodimer of $\mathbf{4 a}$; nor ortho-substituted phthalide of 3ab could be separated from unidentified side-products (entry 9). In addition, whereas products 3ad were, in turn, 
inseparable from trimers of the diyne 2d (entry 11), isochromanones 3ae obtained using 1,7-diyne 2 e could not be separated from unidentified side-products (entry 12). ${ }^{23}$

\section{Side-by-side Solid- and Solution-phase Synthesis of Phthalides via $[2+2+2]-$}

Cycloadditions. In order to face such limitations, we envisaged that, by immobilizing the mono-alkyne component to a solid support, homodimers of type 4 would only form in solution-phase which can be easily separated by simple filtration. Resin-bound crosscycloadducts, on the other hand, could be then selectively cleaved from the solid support by an appropriate reagent.

To this aim, resin-bound propargylamine $\mathbf{1 b}$ was prepared in two steps from BOBA resin (7), which was in turn obtained from readily available Merrifield resin (6) according to the procedure reported by Kobayashi and Aoki (Scheme 3). ${ }^{24,25}$ The loading of the resin 1b was calculated based on the amount of $N$-propargyl- $p$-toluenesulfonamide $(8)$ released upon treatment with either iron chloride or trifluoroacetic acid in dichloromethane (75\% overall yield).

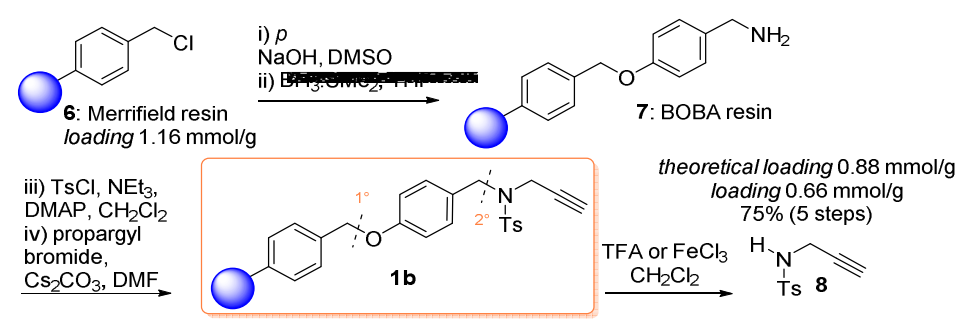

Scheme 3. Preparation of resin $\mathbf{1 b}$ from Merrifield resin.

The use of resin $\mathbf{1 b}$ effectively resulted in the simultaneous generation of benzolactones in both solution and solid phase and the results are summarized in Table 2 . Using $\mathrm{RhCl}\left(\mathrm{PPh}_{3}\right)_{3}$ as catalyst (entries 1-8), the yields and selectivities obtained were 
comparable to those observed in homogeneous media except for the fact that cycloadducts 9 and 4 are naturally easily separated. From the practical point of view, the end of the reaction can be determined by the disappearance of the dyine in the solution phase by TLC, an operation which is not possible in traditional solid-phase synthesis. The release of immobilized products from the resins has been achieved by treatment with trifluoroacetic acid in dichloromethane at room temperature. Thus, released products, derived from immobilized phthalides 9ba-bj, were obtained in good overall yields (40-70\%). Moderate regioselectivity was mostly obtained under conditions used in solution phase experiments, with a preference for the meta regioisomer when the dyine was substituted with a phenyl or ethyl group at the electron deficient triple bond (diynes $\mathbf{2 a}, \mathbf{2 g}, \mathbf{2 h}$ and $\mathbf{2 i}$, entries 1, 5, 6 and 7), regardless of the presence or absence of an internal substituent. Regioselectivity decreased even more when both triple bonds were substituted, such as dyines $\mathbf{2 c}, \mathbf{2} \mathbf{d}$ and $\mathbf{2} \mathbf{j}$ (entries 3, 4 and 8), or when the phenyl substituent was attached to the less electron deficient triple bond ( $\mathbf{2} \mathbf{b}$, entry 2$)$. In general, this pattern of regioselectivity was repeated in the solution-phase products.

Table 2. Side-by-side solid- and solution-phase synthesis of phthalides via [2+2+2]cycloadditions

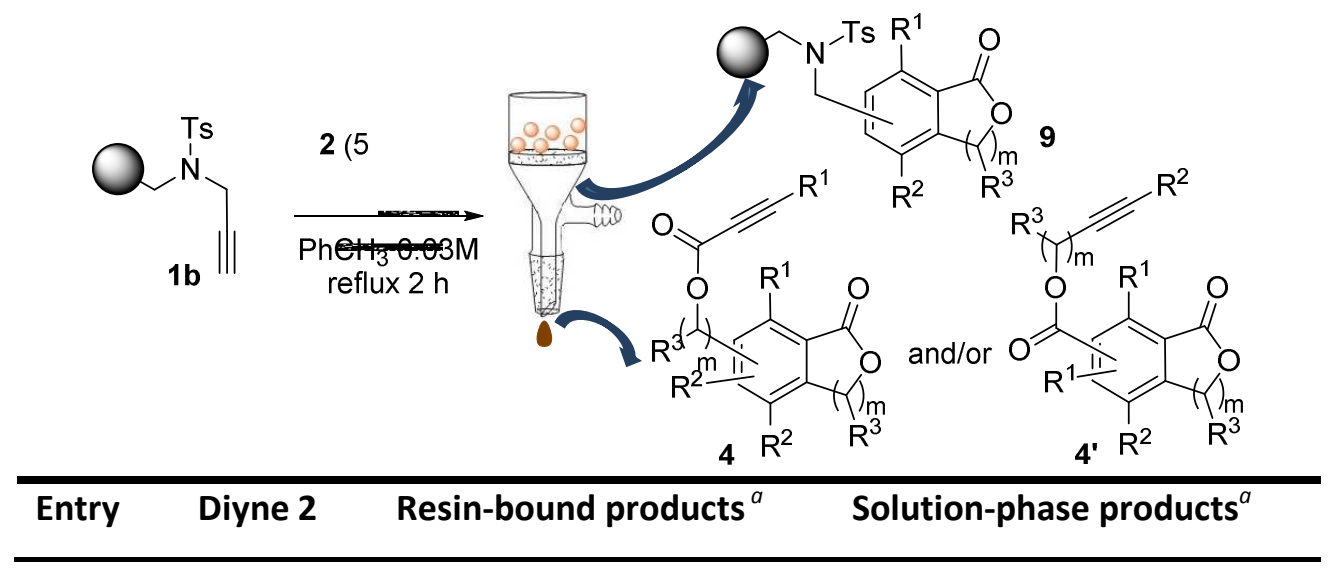


1

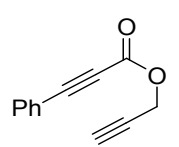

2a

2

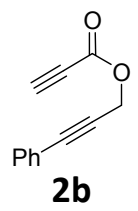

3

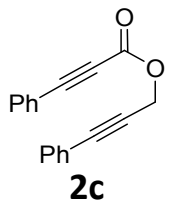

4

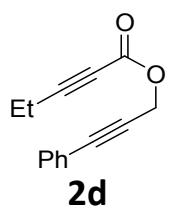

5

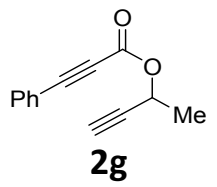

6

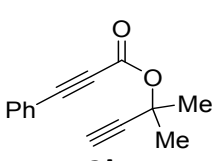

$2 \mathrm{~h}$

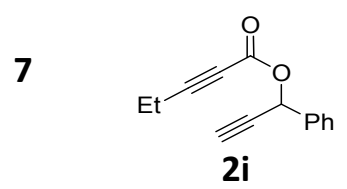

8

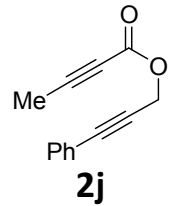

$9^{c}$<smiles>C#CCOC(=O)C#Cc1ccccc1</smiles>

2a

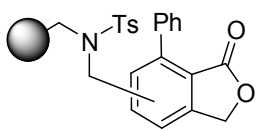

9ba, $70 \%(2.7: 1)$

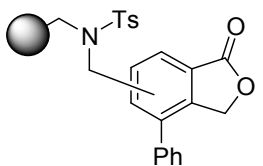

9bb, 40\% (1.3:1)

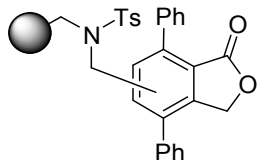

$9 b c, 60 \%(1.2: 1)$

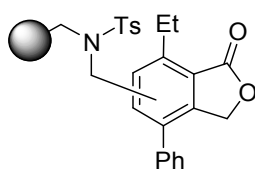

9bd, $60 \%(1.2: 1)$

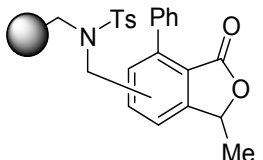

9bg, 70\% (2.7:1)

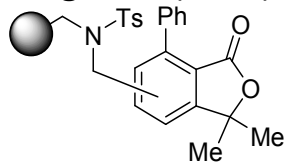

9bh, 60\% (2.5:1)

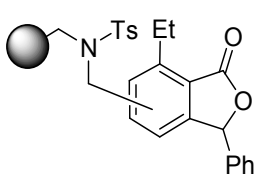

9bi, 55\% (2.8:1)

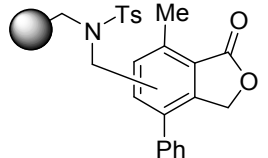

9bj, $63 \%(1.5: 1)$

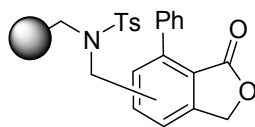

9ba, $50 \%(14: 1)$

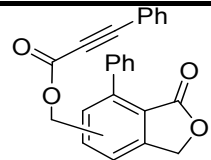

4a, $65 \%(2.2: 1)$

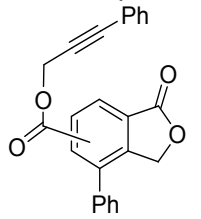

4'b, 30\% (1.3:1)
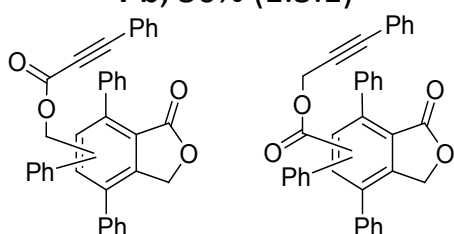

4c, $9 \%(1.4: 1) \quad$ 4'c, $17 \%(3.5: 1)$
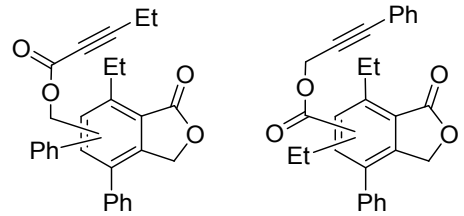

4d, 24\% (4.6:1) 4'd, 25\% (1.7:1)

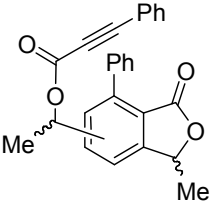

4g, 52\% (3.3:1)
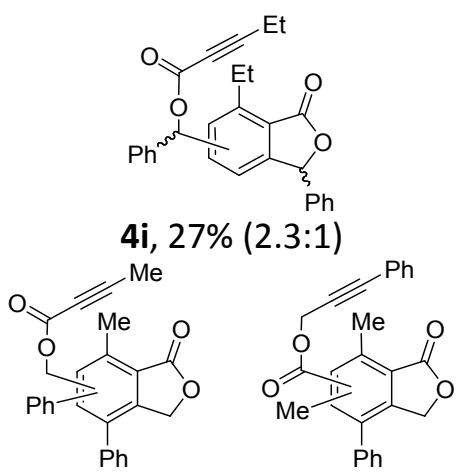

4j, 13\% (2.4:1) 4'j, 15\% (1.2:1)

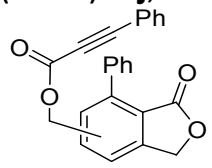

$4 a, 37 \%(13: 1)$ 


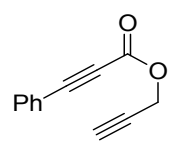

$2 a$

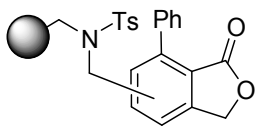

9ba, 52\% (11:1)

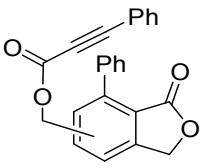

$4 a, 52 \%(13: 1)$

Unless stated, all reactions were performed with 5 equiv of substrates 2 , using $\mathrm{RhCl}\left(\mathrm{PPh}_{3}\right)_{3}$ as catalyst $(5 \mathrm{~mol} \%)$ in toluene $(0.03 \mathrm{M})$, at reflux for 2 hours. Yields of cross-products 9 refer to the amount of resin $\mathbf{1 b}$ used. Yields of homodimers $4 / \mathbf{4}^{\prime}$ 'refer to the amount of substrate $\mathbf{2}$ employed. Yields of crossproducts 9 refer to free phthalides released from resins after TFA treatment (see SI). ${ }^{a}$ Regioisomeric ratio in brackets. ${ }^{b}$ Only DDA products 10h1-2 could be identified in the complex mixture obtained (see text). ${ }^{c}$ Reaction performed with $\mathrm{Cp} * \mathrm{RuCl}(\mathrm{cod})(20 \mathrm{~mol} \%)$ as catalyst, $4 \mathrm{~h}$ at $128^{\circ} \mathrm{C}$. ${ }^{d}$ Conditions as entry 9 , at 100 ${ }^{\circ} \mathrm{C}$ for $6 \mathrm{~h}$.

Also, under these specific experimental reaction conditions it was possible to elucidate a secondary mode of reactivity for diynes $\mathbf{2 c}$ and $\mathbf{2 h}$. Naphthofuranones $\mathbf{1 0 c 1 - 4}$ and 10h1-2 were isolated in the corresponding filtrates in yields ranging from 3.6 to $9 \%$ (Scheme 4A). ${ }^{26} \mathrm{~A}$ possible mechanism for the formation of such cycloisomerization products is depicted in Scheme 4B (particularly for the case of diyne $\mathbf{2 h}$ ) and involves a thermal dehydro-Diels-Alder (DDA) cycloaddition as key step, a reaction that is witnessing a renewed interest by the chemical community. ${ }^{27}$ DDA reaction on substrate $\mathbf{2 h}$ leads to the initial formation of a strained cyclic allene intermediate (11). Product $\mathbf{1 0 h} \mathbf{1}$ is then formed after strain release on intermediate $\mathbf{1 1}$ via hydrogen migration. On the other hand, the formation of $\mathbf{1 0 h} 2$ can be explained via a retro-Diels-Alder reaction on intermediate $\mathbf{1 1}$. This ring-opening reaction produces intermediate $\mathbf{1 2}$ which undergoes a $E-Z$ isomerization and, once again, a DDA cycloaddition. Cyclic allene 14 thus formed delivers product $\mathbf{1 0 h 2}$ after a hydrogen migration.

Scheme 4. Unexpected lactones obtained as side-products. 

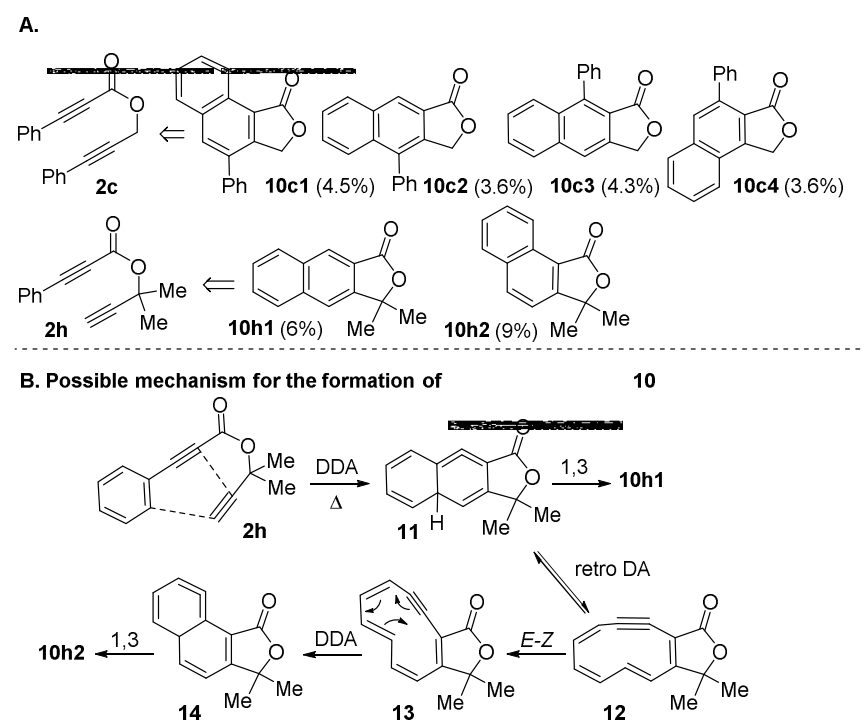

As stated above, a moderate regioselectivity in phthalides 4 and 9 was obtained with Wilkinson's catalyst (Table 2, entries 1-8). To reach an improved regioisomeric ratio, we decided to examine other catalysts. Best results were achieved by using $\mathrm{Cp}^{*} \mathrm{RuCl}(\mathrm{cod})[\mathbf{1 5}$, $\mathrm{Cp} *=\eta^{5}-\mathrm{C}_{5}\left(\mathrm{CH}_{3}\right)_{5}, \operatorname{cod}=1,5$-cyclooctadiene $]$ (Scheme 5). Thus, when the [2+2+2]cycloaddition between resin $\mathbf{1 b}$ and unsymmetrical diyne $\mathbf{2 a}$ was performed in the presence of $20 \mathrm{~mol} \%$ of $\mathrm{Cp}^{*} \mathrm{RuCl}(\mathrm{cod})$ in toluene at reflux, both soluble and immobilized phthalides were obtained with very high regioselectivity, in favor of the meta-substituted aromatic ring (Scheme 5 and Table 2, entries 9 and 10). The regioselectivity of the process can be reasonably explained taking into account the accepted mechanism of the $\mathrm{Cp} * \mathrm{RuCl}-$ catalyzed [2+2+2] cycloaddition of alkynes. ${ }^{18 c, 28}$ After initial ruthenabicycle $\mathbf{A}$ formation between catalyst $\mathbf{1 5}$ and diyne substrate $\mathbf{2 a}$, four modes of approach of the resin $\mathbf{1 b}$ can be conceived for the ensuing [2+2]-cycloaddition step, two ultimately leading to the major product $9 \mathrm{ba1}$ (meta-isomer) and two leading to $\mathbf{9 b a 2}$ (ortho-isomer). The regioselectivity observed can be explained by an initial preferable access of the terminal alkyne to the Ru$\mathrm{C}_{\alpha}$ bond from the less substituted side. This approach would occur, at the same time, regioselectively with the terminal alkyne pointing its terminus toward the chlorine ligand 
thus avoiding steric congestion. Ring opening on brand new $\mathbf{B}$ and eventual reductive elimination would release the catalytically active species $\mathrm{Cp} * \mathrm{RuCl}$ and major phthalide derivative 9ba1. On the other hand, comparable regioselectivity and yields were obtained when the reaction was also carried out in a one-gram scale of resin $\mathbf{1 b}$, proving the reliability of the protocol.
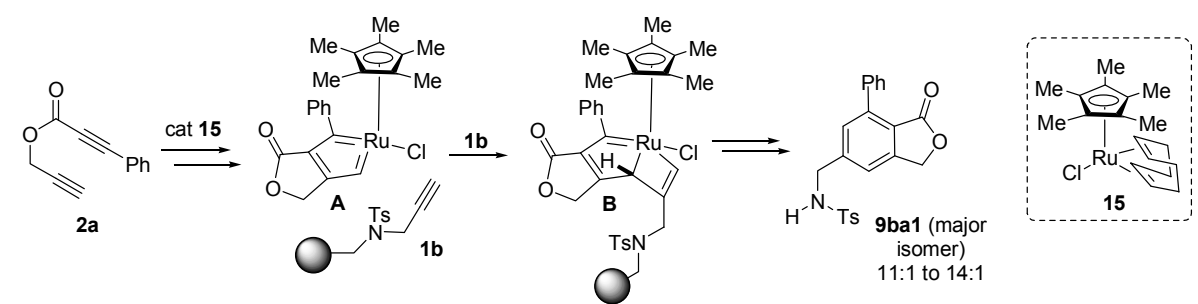

Scheme 5. Regioselective [2+2+2]-cycloaddition of immobilized alkyne $\mathbf{1 b}$

In summary, a complementary approach to simplify [2+2+2]-cycloadditions was explored involving the use of a polymer-supported alkyne. Polysubstituted phthalides were side-by-side assembled both in solution and on a polymer support pointing to a shift in solid-phase chemistry culture in which the filtrate phase becomes waste once the reaction ends. We believe that this proof-of-concept approach can be extended to other reactions such as enyne-alkyne and diyne-sulfonimine ${ }^{29}$ cycloadditions and alkyne-alkyne metathesis, and finds its way into diverse combinatorial strategies.

\section{EXPERIMENTAL SECTION}

\section{Materials and methods}

Unsaturated precursors $\mathbf{1 a}^{30}$ and $\mathbf{2}$ have been previously prepared in the literature. ${ }^{15,18,31}$ All other chemical reagents were purchased from commercial suppliers and 
used without further purification. Solvents were analytical grade or were purified by standard procedures prior to use. Yields were calculated for material judged homogeneous by thin layer chromatography (TLC) and nuclear magnetic resonance $\left({ }^{1} \mathrm{H}\right.$ NMR). All reactions were monitored by thin layer chromatography performed on silica gel $60 \mathrm{~F}_{254}$ precoated aluminum sheets, visualized by a $254 \mathrm{~nm}$ UV lamp, and stained with an ethanolic solution of 4-anisaldehyde. Column flash chromatography was performed using silica gel 60 (230 - 400 mesh). Melting points (M.p.) were taken on an electrothermal melting point apparatus and are uncorrected. Nuclear magnetic resonance spectra were acquired at 300 $\mathrm{MHz}$ for ${ }^{1} \mathrm{H}$ and $75 \mathrm{MHz}$ for ${ }^{13} \mathrm{C}$ using $\mathrm{CDCl}_{3}$ as solvent. Chemical shifts for proton nuclear magnetic resonance spectra are reported in parts per million relative to the signal of tetramethylsilane (TMS) at 0 ppm (internal standard) and coupling constants $(J)$ are reported in hertz $(\mathrm{Hz})$. Chemical shifts for carbon nuclear magnetic resonance $\left({ }^{13} \mathrm{C} \mathrm{NMR}\right)$ spectra are reported in parts per million relative to the center line of the $\mathrm{CDCl}_{3}$ triplet at $77.0 \mathrm{ppm}$. The following abbreviations are used to indicate the multiplicities: $\mathrm{s}=$ singlet, $\mathrm{d}$ $=$ doublet, $\mathrm{t}=$ triplet, $\mathrm{q}=$ quartet, $\mathrm{m}=$ multiplet, quint $=$ quintet, $\mathrm{h}=$ sextet, $\mathrm{br}=$ broad signal. IR spectra were obtained using an FT-IR spectrometer and only partial spectral data are listed. High resolution mass spectra (HRMS) were obtained on a Q-TOF mass spectrometer and detection of the ions was performed in electrospray ionization, positive ion mode. The structure of the products were determined by a combination of spectroscopic methods such as IR, 1D and 2D NMR (including NOE, DEPT, COSY, HSQC and HMBC experiments) and HRMS. NMR signals assignments were based on 2D NMR experiments performed.

\section{Representative procedure for the preparation of phthalides 3 and 4 in solution}


A mixture of $N$-benzyl- $N$-propargyl-p-toluenesulfonamide (1a, 1 mmol, 299 $\mathrm{mg}$ ), diyne 2 (5 mmol, 5 equiv), and Wilkinson catalyst [ $\mathrm{Rh}\left(\mathrm{PPh}_{3}\right)_{3} \mathrm{Cl}, 46 \mathrm{mg}, 0.05$ mmol] in toluene $(33.0 \mathrm{~mL}, 0.03 \mathrm{M})$ was heated at reflux. After approx. 2 hours, the solvent was evaporated under reduced pressure, and the residue was purified by flash column chromatography on silica gel (eluent hexanes/ethyl acetate) to afford the following phthalides 3 and 4 .

\section{Compounds 3aa}

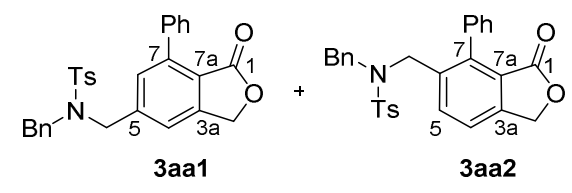

Obtained as a pale yellow liquid consisting of an inseparable mixture of 3aa1 and 3aa2 in an approx. ratio $2.2: 1(0.367 \mathrm{~g}, 0.76 \mathrm{mmol}, 76 \%$ yield $)$. IR (film) $\left(\mathrm{cm}^{-1}\right): 3084$, 3061, 3028, 2926, 1776, 1769, 1454, 1159. ${ }^{1} \mathrm{H} \mathrm{NMR}\left(\mathrm{CDCl}_{3}, 300 \mathrm{MHz}\right)$ : Major isomer 3aa1: $\delta$ 7.79-7.72 (m, 2H, Ts), 7.49-7.29 (m, 7H, Ar-H, Ts), 7.20-7.11 (overlapping s and m, 3H, Ar-H, 4-H), 7.10-7.03 (m, 2H, Ar-H), 7.00 (s, 1H, 6-H), 6.96-6.88 (s, 1H, Ar-H), $5.15(\mathrm{~s}, 2 \mathrm{H}, 3-\mathrm{H}), 4.41\left(\mathrm{~s}, 2 \mathrm{H}, 5-\mathrm{CH}_{2}\right), 4.36(\mathrm{~s}, 2 \mathrm{H}, \mathrm{Bn}), 2.43$ (s, 3H, Ts). Minor isomer 3aa2: $\delta 7.87(\mathrm{~d}, J=8.0,1 \mathrm{H}, 5-\mathrm{H}), 7.61-7.55(\mathrm{~m}, 2 \mathrm{H}, \mathrm{Ts}), 7.49-6.88(\mathrm{~m}, 11 \mathrm{H}, \mathrm{Ar}-\mathrm{H}, 4-\mathrm{H})$, 7.26-7.21 (m, 2H, Ts), 5.20 (s, 2H, 3-H), $4.18\left(\mathrm{~s}, 2 \mathrm{H}, 6-\mathrm{CH}_{2}\right), 4.12$ (s, 2H, Bn), 2.41(s, 3H, Ts). ${ }^{13} \mathrm{C} \mathrm{NMR}\left(\mathrm{CDCl}_{3}, 75 \mathrm{MHz}\right)$ : Major isomer 3aa1: $\delta 169.3$ (C, C-1), 148.1 (C, C-3a), 143.7 (C, Ts), 143.0 (C, C-5), 142.4 (C, C-7), 136.6 (C, Ts), 135.8 (C, C7-Ph), 135.1 (C, Bn), 130.5 (CH, C-6), $129.8(2 \times \mathrm{CH}, \mathrm{Ts}), 129.3(2 \times \mathrm{CH}, \mathrm{Ar}), 128.6(2 \times \mathrm{CH}, \mathrm{Ar}), 128.3$ $(3 \times \mathrm{CH}, \mathrm{Ar}), 127.9(\mathrm{CH}, \mathrm{Ar}), 127.8(2 \times \mathrm{CH}, \mathrm{Ar}), 127.1(2 \times \mathrm{CH}, \mathrm{Ts}), 120.8(\mathrm{C}, \mathrm{C}-7 \mathrm{a})$, $120.4(\mathrm{CH}, \mathrm{C}-4), 68.1\left(\mathrm{CH}_{2}, \mathrm{C}-3\right), 52.3\left(\mathrm{CH}_{2}, \mathrm{Bn}\right), 51.1\left(\mathrm{CH}_{2}, \mathrm{C} 5-\mathrm{CH}_{2}\right), 21.4\left(\mathrm{CH}_{3}, \mathrm{Ts}\right)$. 
Minor isomer 3aa2: $\delta 169.2$ (C, C-1), 146.2 (C, C-3a), 143.4 (C, Ts), 140.3 (C, C-7), 136.3 (C, Ts), 136.1 (C, C-6), 135.0 (C, Bn), 134.4 (CH, C-5), 133.9 (C, Ar), 129.6 (2 × CH, Ts), $128.9(2 \times \mathrm{CH}, \mathrm{Ar}), 128.5(2 \times \mathrm{CH}, \mathrm{Ar}), 128.3(2 \times \mathrm{CH}, \mathrm{Ar}), 128.0(\mathrm{CH}, \mathrm{Ar}), 127.8(2 \times$ CH, Ar), $127.6(\mathrm{CH}, \mathrm{Ar}), 127.0(2 \times \mathrm{CH}, \mathrm{Ts}), 122.7(\mathrm{C}, \mathrm{C}-7 \mathrm{a}), 121,0(\mathrm{CH}, \mathrm{C}-4), 67.9\left(\mathrm{CH}_{2}\right.$, C-3), $52.4\left(\mathrm{CH}_{2}, \mathrm{Bn}\right), 48.0\left(\mathrm{CH}_{2}, \mathrm{C} 6-\mathrm{CH}_{2}\right), 21.4\left(\mathrm{CH}_{3}, \mathrm{Ts}\right)$. HRMS $m / z$ calcd. for $\mathrm{C}_{29} \mathrm{H}_{26} \mathrm{NO}_{4} \mathrm{~S}[\mathrm{M}+\mathrm{H}]^{+}$484.1577, found 484.1573.

\section{Compound 4a1}

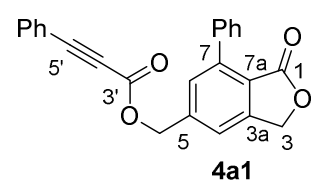

Obtained as a colourless solid (0.322 g, $0.87 \mathrm{mmol}, 35 \%$ yield). M.p.: 128.0-129.0 ${ }^{\circ} \mathrm{C}$. IR (film) $\left(\mathrm{cm}^{-1}\right): 3059,3030,2936,2222,1749,1724,1279,1190,1171,1049 .{ }^{1} \mathrm{H}$ NMR $\left(\mathrm{CDCl}_{3}, 300 \mathrm{MHz}\right): \delta$ 7.63-7.52 (m, 4H, Ar-H), 7.52-7.35 (m, 6H, Ar-H), 7.51 (overlapping s, 1H, 4-H), 7.49 (overlapping s, 1H, 6-H), 5.40 (s, 2H, 1'-H), 5.33 (s, 2H, 3-

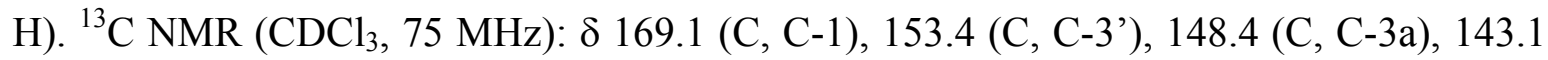
(C, C-7), 141.3 (C, C-5), 135.8 (C, 7-Ph), 132.9 (2 × CH, Ar), 130.8 (CH, Ar), $130.4(\mathrm{CH}$, C-6), $129.4(2 \times \mathrm{CH}, \mathrm{Ar}), 128.52(2 \times \mathrm{CH}, \mathrm{Ar}), 128.49(\mathrm{CH}, \mathrm{Ar}), 127.9(2 \times \mathrm{CH}, \mathrm{Ar}), 121.8$ (C, C-7a), 120.0 (CH, C-4), 119.1 (C, C-6'), 87.5 (C, C-5'), 79.9 (C, C-4'), $68.2\left(\mathrm{CH}_{2}, \mathrm{C}-\right.$ 3), $66.3\left(\mathrm{CH}_{2}, \mathrm{C}-1^{\prime}\right)$. HRMS $m / z$ calcd. for $\mathrm{C}_{24} \mathrm{H}_{17} \mathrm{O}_{4}[\mathrm{M}+\mathrm{H}]^{+}$369.1121, found 369.1129.

\section{Compound 4a2}




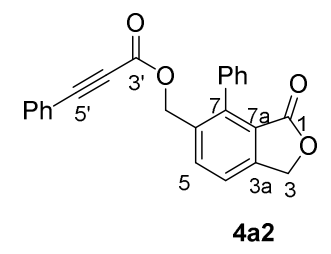

Obtained as a colourless liquid $(0.193 \mathrm{~g}, 0.52 \mathrm{mmol}, 21 \%$ yield $)$. IR (film) $\left(\mathrm{cm}^{-1}\right)$ : 2920, 2849, 2218, 1771, 1707, 1281, 1169. ${ }^{1} \mathrm{H} \mathrm{NMR}\left(\mathrm{CDCl}_{3}, 300 \mathrm{MHz}\right): \delta 7.87(\mathrm{~d}, J=7.9$ Hz, 1H, 5-H), 7.61-7.55 (m, 2H, Ar), 7.52 (d, J=7.9 Hz, 1H, 4-H), 7.50-7.26 (m, 8H, Ar), $5.29(\mathrm{~s}, 2 \mathrm{H}, 3-\mathrm{H}), 5.12\left(\mathrm{~s}, 2 \mathrm{H}, 1{ }^{\prime}-\mathrm{H}\right) .{ }^{13} \mathrm{C} \mathrm{NMR}\left(\mathrm{CDCl}_{3}, 75 \mathrm{MHz}\right): \delta 169.0(\mathrm{C}, \mathrm{C}-1), 153.3$ (C, C-3'), 147.4 (C, C-3a), 142.0 (C, C-7), 134.8 (CH, C-5), 134.6 (C, C-6), 133.6 (C, C7Ph), $132.9(2 \times \mathrm{CH}, \mathrm{Ar}), 130.7(\mathrm{CH}, \mathrm{Ar}), 128.9(2 \times \mathrm{CH}, \mathrm{Ar}), 128.5(2 \times \mathrm{CH}, \mathrm{Ar}), 128.3$ (CH, Ar), $128.0(2 \times \mathrm{CH}, \mathrm{Ar}), 123.3(\mathrm{C}, \mathrm{C}-7 \mathrm{a}), 121.3(\mathrm{CH}, \mathrm{C}-4), 119.2(\mathrm{C}, \mathrm{C}-6$ '), $87.0(\mathrm{C}$, C-5'), 80.0 (C, C-4'), $67.9\left(\mathrm{CH}_{2}, \mathrm{C}-3\right), 64.2\left(\mathrm{CH}_{2}, \mathrm{C}-1^{\prime}\right.$ '). HRMS m/z calcd. for $\mathrm{C}_{24} \mathrm{H}_{17} \mathrm{O}_{4}$ $[\mathrm{M}+\mathrm{H}]^{+}$369.1121, found 369.1124.

\section{Compounds 3ab}

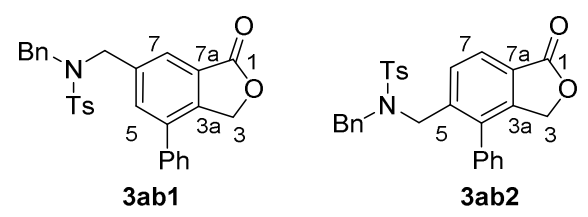

Obtained as a pale yellow liquid consisting of a difficult to separable mixture of 3ab1 and 3ab2 in an approx. ratio 1:1 (0.222 g, $0.46 \mathrm{mmol}, 46 \%$ yield). NMR spectra recorded corresponds to a column chromatography fraction enriched in meta isomer 3ab1. IR (film) $\left(\mathrm{cm}^{-1}\right):$ 3061, 3028, 2922, 1765, 1339, 1159. ${ }^{1} \mathrm{H}$ NMR ( $\left.\mathrm{CDCl}_{3}, 300 \mathrm{MHz}\right):$ meta isomer 3ab1: $\delta$ 7.79-7.74 (m, 2H, Ts), 7.52-7.38 (m, 5H, Ar, 5-H, 7-H), 7.36-7.30 (m, 2H, Ts), 7.30-7.25 (m, 2H, Ar), 7.22-7.14 (m, 3H, Ar), 7.14-7.06 (m, 2H, Ar), 5.31 (s, 2H, 3- 
H), $4.43\left(\mathrm{~s}, 2 \mathrm{H}, 6-\mathrm{CH}_{2}\right), 4.36(\mathrm{~s}, 2 \mathrm{H}, \mathrm{Bn}), 2.44$ (s, 3H, Ts). ortho Isomer 3ab2: $\delta$ 7.81-7.56 (overlapping signal, 1H, 7-H), 7.66-7.58 (overlapping signals, 3H, Ts, 6-H), 7.52-7.08 (m, 8H, Ar, Ts), 6.94-6.88 (m, 4H, Ar), 4.91 (s, 2H, 3-H), 4.25 (s, 2H, 5- $\left.\mathrm{CH}_{2}\right), 4.19$ (s, 2H, Bn),

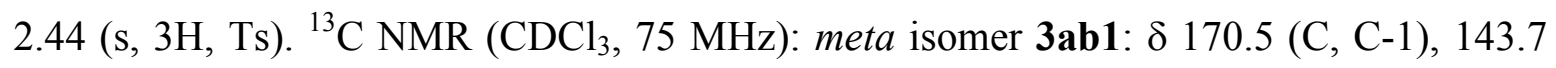
(C, Ts), 143.4 (C, C-3a), 138.5 (C, C-6), 137.0 (C, C-4), 136.9 (C, Ar), 136.8 (C, Ts), 135.2 (C, Ar), $133.9(\mathrm{CH}, \mathrm{C}-5), 129.8(2 \times \mathrm{CH}, \mathrm{Ts}), 129.0(2 \times \mathrm{CH}, \mathrm{Ar}), 128.5(3 \times \mathrm{CH}, \mathrm{Ar})$, $128.4(2 \times \mathrm{CH}, \mathrm{Ar}), 127.7(\mathrm{CH}, \mathrm{Ar}), 127.5(2 \times \mathrm{CH}, \mathrm{Ar}), 127.1(2 \times \mathrm{CH}, \mathrm{Ts}), 126.3(\mathrm{C}, \mathrm{C}-$ 7a), 123.9 (CH, C-7), $69.4\left(\mathrm{CH}_{2}, \mathrm{C}-3\right), 51.9\left(\mathrm{CH}_{2}, \mathrm{Bn}\right), 50.7\left(\mathrm{CH}_{2}, \mathrm{C} 6-\mathrm{CH}_{2}\right), 21.4\left(\mathrm{CH}_{3}\right.$, Ts). ortho Isomer 3ab2: $\delta 170.8$ (C, C-1), 145.8 (C-3a), 143.5 (C, Ts), 140.7 (C, C-5), 136.4 (C, Ts), 135.5 (C, C-4), 134.9 (C, Ar), 134.8 (C, Ar), 129.7 (CH, C-6), 128.5 (2 × $\mathrm{CH}, \mathrm{Ar}), 128.3(2 \times \mathrm{CH}, \mathrm{Ar}), 127.1(2 \times \mathrm{CH}, \mathrm{Ts}), 124.6(\mathrm{C}, \mathrm{C}-7 \mathrm{a}), 124.5(\mathrm{CH}, \mathrm{C}-7), 69.0$ $\left(\mathrm{CH}_{2}, \mathrm{C}-3\right), 52.2\left(\mathrm{CH}_{2}, \mathrm{Bn}\right), 48.1\left(\mathrm{CH}_{2}, \mathrm{C} 5-\mathrm{CH}_{2}\right), 21.4\left(\mathrm{CH}_{3}, \mathrm{Ts}\right)$. Unassigned signals: $\delta$ 128.9, 128.2, 127.8. HRMS $\mathrm{m} / z$ calcd. for $\mathrm{C}_{29} \mathrm{H}_{25} \mathrm{KNO}_{4} \mathrm{~S}[\mathrm{M}+\mathrm{K}]^{+}$522.1136, found 522.1135.

\section{Compounds 4'b}

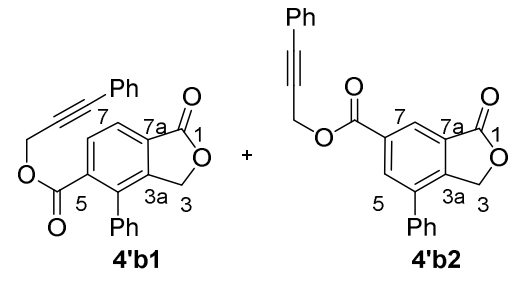

Obtained as a yellow liquid consisting of a difficult to separate mixture of $\mathbf{4}^{\prime} \mathbf{b} \mathbf{1}$ and 4'b2 in an approx. ratio 2:1 (0.322 g, $0.87 \mathrm{mmol}, 35 \%$ yield). IR (film) $\left(\mathrm{cm}^{-1}\right): 3059,3024$, 
2232, 1769, 1732, 1217. ${ }^{1} \mathrm{H}$ NMR $\left(\mathrm{CDCl}_{3}, 300 \mathrm{MHz}\right)$ : Major isomer 4'b1: $\delta 8.10(\mathrm{~d}, J=$ $7.9 \mathrm{~Hz}, 1 \mathrm{H}, 6-\mathrm{H}), 7.98$ (d, $J=7.9,1 \mathrm{H}, 7-\mathrm{H}), 7.57-7.24$ (m, 10H, Ar-H), 5.14 (s, 2H, 3-H), $4.90\left(\mathrm{~s}, 2 \mathrm{H}, 3^{\prime}-\mathrm{H}\right)$. Minor isomer 4'b2: $\delta 8.65(\mathrm{~d}, J=1.2 \mathrm{~Hz}, 1 \mathrm{H}, 7-\mathrm{H}), 8.46(\mathrm{~d}, J=1.3,1 \mathrm{H}$, 5-H), 7.57-7.24 (m, 10H, Ar-H), 5.46 (s, 2H, 3-H), 5.22 (s, 2H, 3'-H). ${ }^{13} \mathrm{C}$ NMR $\left(\mathrm{CDCl}_{3}\right.$, 75 MHz): Major isomer 4'b1: $\delta 170.0$ (C, C-1), 166.0 (C, C-1'), 146.2 (C, C-3a), 137.7 (C, C-4), 135.8 (C, Ar), 135.0 (C, C-5), 131.7 (2 × CH, Ar), 131.0 (CH, C-6), $128.74(2 \times \mathrm{CH}$, Ar), 128.3 (C, C-7a), $128.2(2 \times \mathrm{CH}, \mathrm{Ar}), 127.62(2 \times \mathrm{CH}, \mathrm{Ar}), 124.4(\mathrm{CH}, \mathrm{C}-7), 121.84(\mathrm{C}$, Ar), 86.7 (C, C-5'), 81.9 (C, C-4'), $69.4\left(\mathrm{CH}_{2}, \mathrm{C}-3\right), 53.6\left(\mathrm{CH}_{2}, \mathrm{C}-3\right.$ '). Minor isomer 4'b2:

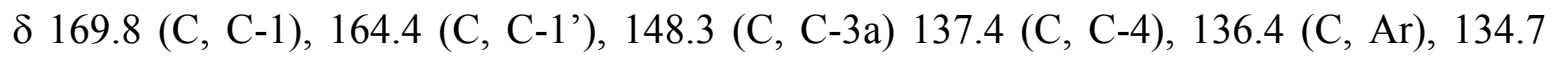
(CH, C-5), 131.8 (2 × CH, Ar), 127.0 (C, C-7a), $126.0(\mathrm{CH}, \mathrm{C}-7), 121.79$ (C, Ar), 87.0 (C, C-5'), 82.3 (C, C-4'), $69.6\left(\mathrm{CH}_{2}, \mathrm{C}-3\right), 53.9\left(\mathrm{CH}_{2}, \mathrm{C}-3\right.$ '). Unassigned signals: $\delta$ 129.6, $129.2,128.82,128.76,128.65,128.4,127.64,127.1$. HRMS $m / z$ calcd. for $\mathrm{C}_{24} \mathrm{H}_{17} \mathrm{O}_{4}[\mathrm{M}+$ $\mathrm{H}]^{+}$369.1121, found 369.1122 .

\section{Compounds 3ac}
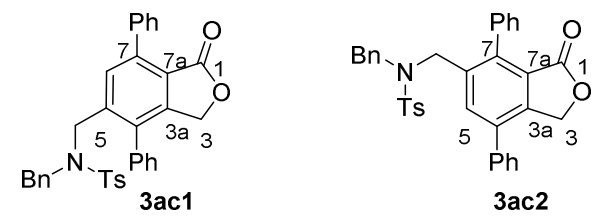

Obtained as a pale yellow solid consisting of an inseparable mixture of 3ac1 and 3ac2 in an approx. ratio $1.2: 1$ (0.358 g, $0.64 \mathrm{mmol}, 64 \%$ yield). M.p.: $159.6-165.7{ }^{\circ} \mathrm{C}$. IR (film) $\left(\mathrm{cm}^{-1}\right): 3059,3026,2924,1763,1447,1340,1159 .{ }^{1} \mathrm{H}$ NMR $\left(\mathrm{CDCl}_{3}, 300 \mathrm{MHz}\right)$ : Major isomer 3ac1: $\delta$ 7.63-6.93 (m, 19 H, Ar-H, Ts), 7.34 (overlapping s, 1H, 6-H), 4.88 (s, 2H, 3-H), $4.28\left(\mathrm{~s}, 2 \mathrm{H}, 5-\mathrm{CH}_{2}\right), 4.24(\mathrm{~s}, 2 \mathrm{H}, \mathrm{Bn}), 2.37$ (s, 3H, Ts). Minor isomer 3ac2: $\delta$ 
$7.70(\mathrm{~s}, 1 \mathrm{H}, 5-\mathrm{H}), 7.63-6.93$ (m, 19H, Ar-H, Ts), 5.27 (s, 2H, 3-H), 4.23 (s, 2H, 6-CH , $^{2}$ 4.18 (s, 2H, Bn), 2.38 (s, 3H, Ts). ${ }^{13} \mathrm{C}$ NMR $\left(\mathrm{CDCl}_{3}, 75 \mathrm{MHz}\right)$ : Major isomer 3ac1: $\delta 169.5$ (C, C-3), 147.2 (C, C-3a), 140.4 (C, C-5), 134.1 (C, C-4), 130.9 (CH, C-6), 120.5 (C, C7a), $67.8\left(\mathrm{CH}_{2}, \mathrm{C}-3\right), 52.3\left(\mathrm{CH}_{2}, \mathrm{Bn}\right), 48.0\left(\mathrm{CH}_{2}, \mathrm{C} 5-\mathrm{CH}_{2}\right), 21.4\left(\mathrm{CH}_{3}\right.$, Ts $)$. Minor isomer 3ac2: $\delta 169.2$ (C, C-3), 143.7 (C, C-3a), 139.1 (C, C-7), 137.1 (C, Ar), 136.9 (C, C-6), 135.6 (C, C-4), 133.8 (CH, C-5), 123.3 (C, C-7a), $67.9\left(\mathrm{CH}_{2}, \mathrm{C}-3\right), 52.7\left(\mathrm{CH}_{2}, \mathrm{Bn}\right), 48.2$ $\left(\mathrm{CH}_{2}, \mathrm{C} 6-\mathrm{CH}_{2}\right), 21.4\left(\mathrm{CH}_{3}, \mathrm{Ts}\right)$. Unassigned signals: $\delta 143.5,143.4,143.3,141.4,136.5$, $136.2,135.8,135.2,135.0,134.7,129.68,129.66,129.4,129.1,128.9,128.6,128.5,128.4$ 128.32, 128.26, 127.9, 127.82, 127.76, 127.7, 127.6, 127.1, 126.97. HRMS m/z calcd. for $\mathrm{C}_{35} \mathrm{H}_{30} \mathrm{NO}_{4} \mathrm{~S}[\mathrm{M}+\mathrm{H}]^{+}$560.1890, found 560.1898 .

\section{Compounds 4c and 4'c}

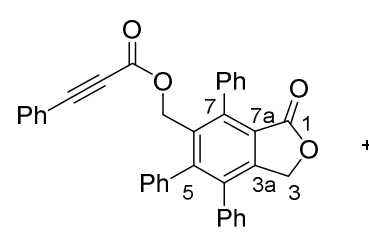

$4 c 1$

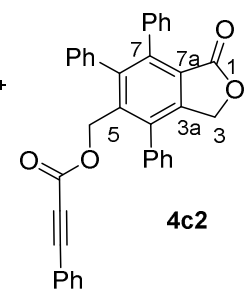

Obtained as a pale yellow liquid consisting of an inseparable mixture of compounds 4c1/4c2 and 4'c1/4'c2 in an approx. ratio 2:1:3.4:1 (0.403 g, $0.77 \mathrm{mmol}, 31 \%$ yield). IR (film) $\left(\mathrm{cm}^{-1}\right): 3057,3022,2218,1771,1738,1711,1194 .{ }^{1} \mathrm{H}$ NMR $\left(\mathrm{CDCl}_{3}, 300 \mathrm{MHz}\right): \delta$ 7.63-7.01 (m, 80H, Ar-H), 5.17 (s, 2H, 3-H), $5.13(\mathrm{~s}, 2 \mathrm{H}, 3-\mathrm{H}), 5.10$ (s, 2H, 3-H), 5.08 (s, 2H, 3-H), 4.84 (s, 2H, 1'-H), 4.82 (s, 2H, 1'-H), 4.45 (s, 2H, 3'-H), 4.42 (s, 2H, 3'-H). ${ }^{13} \mathrm{C}$ $\mathrm{NMR}\left(\mathrm{CDCl}_{3}, 75 \mathrm{MHz}\right): \delta 169.2,169.1,168.90,168.87,166.7,166.6,152.7,152.6,148.7$, $147.3,146.8,145.6,145.3,145.2,144.1,143.1,141.1,141.0,140.8,138.9,138.7,137.8$, 
$136.9,136.8,136.5,136.44,136.40,136.2,136.0,135.7,135.3,135.2,134.8,134.7,134.1$, 133.9, 133.51, 133.46, 133.1, 132.9, 131.7, 130.7, 130.6, 130.1, 130.00, 129.96, 129.8, $129.7,129.6,129.1,129.0,128.9,128.8,128.7,128.65,128.60,128.5,128.4,128.2,128.1$, $127.9,127.7,127.64,127.56,127.53,127.48,127.4,127.3,127.2,127.1,123.7,123.2$, $122.5,122.0,121.95,121.92,119.4,119.3,86.4,86.3,82.3,81.8,81.6,80.1,79.9,68.1$, $68.0,67.8,67.7,62.9,62.7,53.13,53.06$. HRMS $m / z$ calcd. for $\mathrm{C}_{36} \mathrm{H}_{25} \mathrm{O}_{4}[\mathrm{M}+\mathrm{H}]^{+}$ 521.1747 , found 521.1731 .

\section{Compounds 3ad}

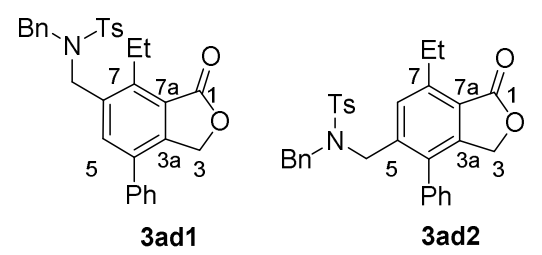

Obtained as a pale yellow liquid consisting of an inseparable mixture of 3ad1 and 3ad2 in an approx. ratio 1.1:1 and trimers $5 d(0.409 \mathrm{~g}, 0.80 \mathrm{mmol}, 80 \%$ yield). IR (film) $\left(\mathrm{cm}^{-1}\right): 3061,3028,2970,2934,1759,1730,1342,1159 .{ }^{1} \mathrm{H}$ NMR $\left(\mathrm{CDCl}_{3}, 300 \mathrm{MHz}\right)$ : Major isomer 3ad1: $\delta$ 7.81-7.74 (m, 2H, Ts), 7.52-6.86 (m, 13H, Ar, Ts, 5-H), $5.21(\mathrm{~s}, 2 \mathrm{H}$, 3-H), 4.50 (s, 2H, 6- $\left.\mathrm{CH}_{2}\right), 4.37$ (s, 2H, Bn), 2.96 (q, $\left.J=7.4 \mathrm{~Hz}, 2 \mathrm{H}, 7-\mathrm{Et}\right), 2.42$ (s, 3H, Ts), $1.02(\mathrm{t}, J=7.5 \mathrm{~Hz}, 3 \mathrm{H}, 7-\mathrm{Et})$. Minor isomer 3ad2: $\delta$ 7.66-7.59 (m, 2H, Ts), 7.52-6.86 (m, 13H, Ar, Ts, 6-H), 4.84 (s, 2H, 3-H), 4.23 (s, 2H, 5- $\left.\mathrm{CH}_{2}\right), 4.21$ (s, 2H, Bn), 3.01 (q, J= 7.5 $\mathrm{Hz}, 2 \mathrm{H}, 7-\mathrm{Et}), 2.43$ (s, 3H, Ts), 1.21 (t, $J=7.5 \mathrm{~Hz}, 3 \mathrm{H}, 7-\mathrm{Et}) .{ }^{13} \mathrm{C} \mathrm{NMR}\left(\mathrm{CDCl}_{3}, 75 \mathrm{MHz}\right)$ : Major isomer 3ad1: $\delta 170.3$ (C, C-1), 144.1 (C, C-3a), 143.6 (C, Ts), 143.1 (C, C-7), 137.2 (C, Ph), 136.6 (C, Ts), 135.3 (C, Bn), 135.2 (C, C-6), 134.1 (CH, C-5), 133.8 (C, C-4), 
$129.8(2 \times \mathrm{CH}, \mathrm{Ts}), 127.1(2 \times \mathrm{CH}, \mathrm{Ts}), 123.1(\mathrm{C}, \mathrm{C}-7 \mathrm{a}), 68.0\left(\mathrm{CH}_{2}, \mathrm{C}-3\right), 51.9\left(\mathrm{CH}_{2}, \mathrm{Bn}\right)$, $47.4\left(\mathrm{CH}_{2}, \mathrm{C} 6-\mathrm{CH}_{2}\right), 21.4\left(\mathrm{CH}_{3}, \mathrm{Ts}\right), 19.2\left(\mathrm{CH}_{2}, \mathrm{C} 7-\mathrm{Et}\right), 14.8\left(\mathrm{CH}_{3}, \mathrm{C} 7-\mathrm{Et}\right)$. Minor isomer 3ad2: $\delta 170.6$ (C, C-1), 146.4 (C-3a), 144.6 (C, C-7), 143.5 (C, Ts), 140.2 (C, C-5), 136.6 (C, Ts), 135.02 (C, Bn), 134.98 (C, Ph), 132.9 (C, C-4), 129.7 (2 × CH, Ts), 129.2 (CH, C6), $127.0(2 \times \mathrm{CH}, \mathrm{Ts}), 121.4(\mathrm{C}, \mathrm{C}-7 \mathrm{a}), 68.2\left(\mathrm{CH}_{2}, \mathrm{C}-3\right), 52.2\left(\mathrm{CH}_{2}, \mathrm{Bn}\right), 48.1\left(\mathrm{CH}_{2}, \mathrm{C} 5-\right.$ $\left.\mathrm{CH}_{2}\right), 23.8\left(\mathrm{CH}_{2}, \mathrm{C} 7-\mathrm{Et}\right), 21.3\left(\mathrm{CH}_{3}, \mathrm{Ts}\right), 14.8\left(\mathrm{CH}_{3}, 7-\mathrm{Et}\right)$. Unassigned signals: $\delta 128.84$, 128.79, 128.3, 128.2, 127.5. HRMS $m / z$ calcd. for $\mathrm{C}_{31} \mathrm{H}_{30} \mathrm{NO}_{4} \mathrm{~S}[\mathrm{M}+\mathrm{H}]^{+}$512.1890, found 512.1881 .

\section{Compounds 4d}
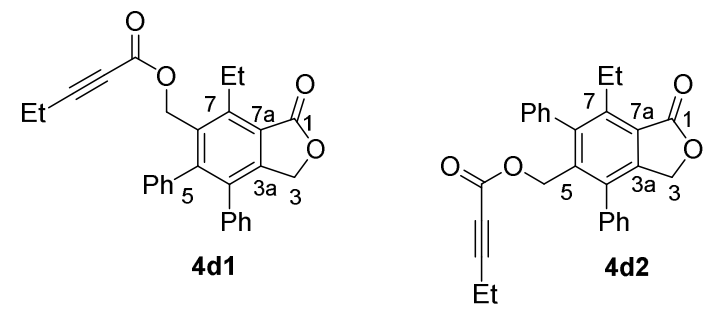

Obtained as a colourless liquid consisting of an inseparable mixture of $\mathbf{4 d \mathbf { 1 }}$ and $\mathbf{4 d \mathbf { 2 }}$ in an approx. ratio 5:1 (0.222 $\mathrm{g}, 0.52 \mathrm{mmol}, 21 \%$ yield). IR (film) $\left(\mathrm{cm}^{-1}\right): 3059,2980,2938$, 2235, 1759, 1713, 1244. ${ }^{1} \mathrm{H} \mathrm{NMR}\left(\mathrm{CDCl}_{3}, 300 \mathrm{MHz}\right)$ : Major isomer 4d1: $\delta$ 7.25-7.13 (m, 6H, Ar-H), 7.08-7.00 (m, 2H, Ar-H), 6.99-6.93 (m, 2H, Ar-H), 5.043 (s, 2H, 3-H), 5.039 (s, 2H, 6- $\mathrm{CH}_{2}$ ), 3.29 (q, $\left.J=7.5 \mathrm{~Hz}, 2 \mathrm{H}, 7-\mathrm{Et}\right), 2.34$ (q, $J=7.5 \mathrm{~Hz}, 2 \mathrm{H}, 6$ '-H), 1.35 (t, $J=7.4$ Hz, 7-Et), 1.20 (t, $\left.J=7.5 \mathrm{~Hz}, 7^{\prime}-\mathrm{H}\right)$. Minor isomer 4d2: $\delta$ 7.50-7.39 (m, 6H, Ar-H), 7.35$7.28(\mathrm{~m}, 2 \mathrm{H}, \mathrm{Ar}-\mathrm{H}), 7.27-7.19(\mathrm{~m}, 2 \mathrm{H}, \mathrm{Ar}-\mathrm{H}), 5.03(\mathrm{~s}, 2 \mathrm{H}, 3-\mathrm{H}), 4.65$ (s, 2H, 1'-H), 2.88 (q, $J=7.5 \mathrm{~Hz}, 2 \mathrm{H}, 7-\mathrm{Et}), 2.32$ (q, $\left.J=7.5 \mathrm{~Hz}, 2 \mathrm{H}, 6^{\prime}-\mathrm{H}\right), 1.20$ (t, $\left.J=7.5 \mathrm{~Hz}, 3 \mathrm{H}, 7^{\prime}-\mathrm{H}\right), 1.08$ (t, 
$J=7.5 \mathrm{~Hz}, 3 \mathrm{H}, 7-\mathrm{Et}) .{ }^{13} \mathrm{C} \mathrm{NMR}\left(\mathrm{CDCl}_{3}, 75 \mathrm{MHz}\right)$ : Major isomer 4d1: $\delta 170.4(\mathrm{C}, \mathrm{C}-1)$, 153.0 (C, C-3’), 148.7 (C, C-5), 147.0 (C, C-3a), 146.1 (C, C-7), 137.4 (C, Ar), 136.3 (C, Ar), 134.4 (C, C-4), 132.4 (C, C-6), $129.6(2 \times \mathrm{CH}, \mathrm{Ar}), 128.9(2 \times \mathrm{CH}, \mathrm{Ar}), 128.1(2 \times$ CH, Ar), 127.7 (2 × CH, Ar), $127.4(\mathrm{CH}, \mathrm{Ar}), 127.3(\mathrm{CH}, \mathrm{Ar}), 122.2$ (C, C-7a), 91.1 (C, C5'), 71.9 (C, C-5'), $68.3\left(\mathrm{CH}_{2}, \mathrm{C}-3\right), 61.8\left(\mathrm{CH}_{2}, \mathrm{C}-1\right.$ '), $20.8\left(\mathrm{CH}_{2}, 7-\mathrm{Et}\right), 15.7\left(\mathrm{CH}_{3}, 7-\mathrm{Et}\right)$, $12.4\left(\mathrm{CH}_{3}, \mathrm{C}-7^{\prime}\right), 12.3\left(\mathrm{CH}_{2}, \mathrm{C}^{\prime} 6^{\prime}\right)$. Minor isomer 4d2: $\delta 170.5(\mathrm{C}, \mathrm{C}-1), 152.5\left(\mathrm{C}, \mathrm{C}-3^{\prime}\right)$, 145.8 (C, C-3a), 145.0 (C, C-6), 143.9 (C, C-7), 137.0 (C, Ar), 136.4 (C, C-5), 135.8 (C, C4), 135.4 (C, Ar), $129.4(2 \times \mathrm{CH}, \mathrm{Ar}), 128.8(2 \times \mathrm{CH}, \mathrm{Ar}), 128.5(2 \times \mathrm{CH}, \mathrm{Ar}), 128.4(\mathrm{CH}$, Ar), $128.2(2 \times \mathrm{CH}, \mathrm{Ar}), 127.8$ (CH, Ar), 123.0 (C, C-7a), 90.7 (C, C-5'), 71.8 (C, C-4'), $68.3\left(\mathrm{CH}_{2}, \mathrm{C}-3\right), 62.4\left(\mathrm{CH}_{2}, \mathrm{C}-1\right.$ '), $21.6\left(\mathrm{CH}_{2}, 7-\mathrm{Et}\right), 15.4\left(\mathrm{CH}_{3}, 7-\mathrm{Et}\right), 12.4\left(\mathrm{CH}_{3}, \mathrm{C}-{ }^{\prime}{ }^{\prime}\right)$, 12.3 $\left(\mathrm{CH}_{2}, \mathrm{C}-6^{\prime}\right)$. HRMS $m / z$ calcd. for $\mathrm{C}_{28} \mathrm{H}_{25} \mathrm{O}_{4}[\mathrm{M}+\mathrm{H}]^{+} 425.1747$, found 425.1753 .

\section{Compounds 4'd}
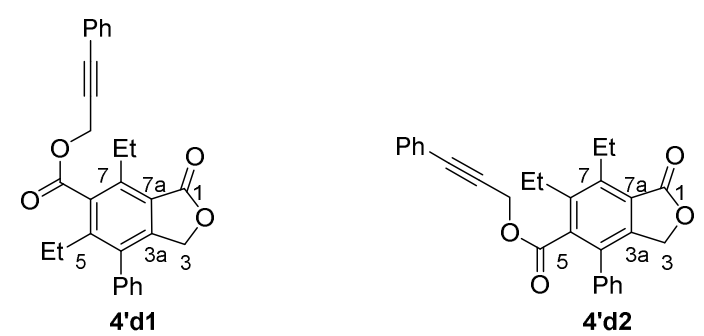

Obtained as a pale yellow liquid consisting of an inseparable mixture of 4'd1 and 4'd2 in an approx. ratio 1.5:1 (0.212 g, $0.50 \mathrm{mmol}, 20 \%$ yield). IR (film) $\left(\mathrm{cm}^{-1}\right): 2972$, 2936, 1761, 1738, 1198, 1028. ${ }^{1} \mathrm{H} \mathrm{NMR}\left(\mathrm{CDCl}_{3}, 300 \mathrm{MHz}\right)$ : Major isomer 4'd1: $\delta$ 7.527.20 (m, 10H, Ar-H), 5.21 (s, 2H, 3'-H), 4.89 (s, 2H, 3-H), 3.13 (q, $J=7.5 \mathrm{~Hz}, 2 \mathrm{H}, 7-\mathrm{Et})$, 2.61 (q, $J=7.5 \mathrm{~Hz}, 2 \mathrm{H}, 5-\mathrm{Et}), 1.33$ (t, $J=7.5 \mathrm{~Hz}, 3 \mathrm{H}, 7-\mathrm{Et}), 1.02$ (t, $J=7.5 \mathrm{~Hz}, 3 \mathrm{H}, 5$-Et). 
Minor isomer 4'd2: $\delta$ 7.52-7.20 (m, 10H, Ar-H), 5.04 (s, 2H, 3-H), 4.81 (s, 2H, 3'-H), 3.23 (q, $J=7.5 \mathrm{~Hz}, 2 \mathrm{H}, 7-\mathrm{Et}), 2.81(\mathrm{q}, J=7.5 \mathrm{~Hz}, 2 \mathrm{H}, 6-\mathrm{Et}), 1.27$ (t, $J=7.5 \mathrm{~Hz}, 3 \mathrm{H}, 7-\mathrm{Et}), 1.26$ $(\mathrm{t}, J=7.5 \mathrm{~Hz}, 3 \mathrm{H}, 6-\mathrm{Et}) .{ }^{13} \mathrm{C} \mathrm{NMR}\left(\mathrm{CDCl}_{3}, 75 \mathrm{MHz}\right)$ : Major isomer 4'd1: $\delta 170.1(\mathrm{C}, \mathrm{C}-1)$, 168.2 (C, C-1'), 147.9 (C, C-3a), 145.4 (C, C-5), 142.1 (C, C-7), 135.3 (C, Ar), 134.5 (C, C-4), $131.6(2 \times \mathrm{CH}, \mathrm{Ar}), 128.9(2 \times \mathrm{CH}, \mathrm{Ar}), 128.7(2 \times \mathrm{CH}, \mathrm{Ar}), 128.4(\mathrm{CH}, \mathrm{Ar}), 128.2(2$ $\times \mathrm{CH}, \mathrm{Ar}), 121.8\left(\mathrm{C}, \mathrm{C}-6^{\prime}\right), 120.5$ (C, C-7a), 87.0 (C, C-5'), 82.0 (C, C-4'), 68.3 (CH, C3), $53.5\left(\mathrm{CH}_{2}, \mathrm{C}-3^{\prime}\right), 24.3\left(\mathrm{CH}_{2}, \mathrm{C} 5-\mathrm{Et}\right), 22.3\left(\mathrm{CH}_{2}, \mathrm{C} 7-\mathrm{Et}\right), 15.6\left(\mathrm{CH}_{3}, \mathrm{C} 7-\mathrm{Et}\right), 15.3\left(\mathrm{CH}_{3}\right.$, C5-Et). Minor isomer 4'd2: $\delta 170.3$ (C, C-1), 167.8 (C, C-1'), 143.9 (C, C-7), 143.8 (C, C3a), 140.6 (C, C-6), 138.4 (C, C-5), 135.3 (C, Ar), 132.0 (C, C-4), 131.7 (2 × CH, Ar), $128.8(\mathrm{CH}, \mathrm{Ar}), 128.7(\mathrm{CH}, \mathrm{Ar}), 128.5(2 \times \mathrm{CH}, \mathrm{Ar}), 128.25(2 \times \mathrm{CH}, \mathrm{Ar}), 128.20(2 \times \mathrm{CH}$, Ar), 123.7 (C, C-7a), 121.9 (C, C-6'), 86.7 (C, C-5'), 81.8 (C, C-4'), 67.9 ( $\left.\mathrm{CH}_{2}, \mathrm{C}-3\right), 53.2$ $\left(\mathrm{CH}_{2}, \mathrm{C}-3\right.$ ') $23.0\left(\mathrm{CH}_{2}, \mathrm{C} 6-\mathrm{Et}\right), 19.9\left(\mathrm{CH}_{2}, \mathrm{C} 7-\mathrm{Et}\right), 15.8\left(\mathrm{CH}_{3}, \mathrm{C} 6-\mathrm{Et}\right), 15.5\left(\mathrm{CH}_{3}, \mathrm{C} 7-\mathrm{Et}\right)$. HRMS $m / z$ calcd. for $\mathrm{C}_{28} \mathrm{H}_{25} \mathrm{O}_{4}[\mathrm{M}+\mathrm{H}]^{+}$425.1747, found 425.1747 .

\section{Compounds 5d}

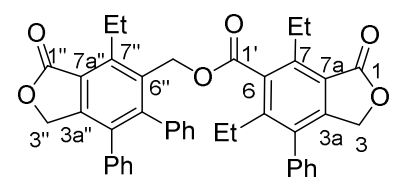

Obtained as a pale yellow liquid consisting of an inseparable mixture of trimers $\mathbf{5 d}$ in an approx. ratio $2: 1(0.178 \mathrm{~g}, 0.28 \mathrm{mmol}, 17 \%$ yield $) . \mathrm{IR}($ film $)\left(\mathrm{cm}^{-1}\right): 3022,2972,2936$, 2876, 1759, 1728, 1028. ${ }^{1} \mathrm{H}$ NMR $\left(\mathrm{CDCl}_{3}, 300 \mathrm{MHz}\right)$ : Only major isomer could be analysed, $\delta$ 7.53-6.85 (m, 15H, Ar-H), 5.32 (s, 2H, 3'-H), 5.04 (s, 2H, 3'’-H), 4.87 (s, 2H, 3-H), 3.34 (q, $J=7.4 \mathrm{~Hz}, 2 \mathrm{H}, 7$ ' '-Et), 2.95 (q, $J=7.5 \mathrm{~Hz}, 2 \mathrm{H}, 7-\mathrm{Et}), 2.48$ (q, $J=7.5 \mathrm{~Hz}$, 
2H, 5-Et), 1.36 (t, $J=7.3 \mathrm{~Hz}, 3 \mathrm{H}, 7^{\prime}$ '-H), 1.19 (t, $\left.J=7.4 \mathrm{~Hz}, 3 \mathrm{H}, 7^{\prime}-\mathrm{H}\right), 0.88$ (t, $J=7.6 \mathrm{~Hz}$, $3 \mathrm{H}, 5-\mathrm{H}) .{ }^{13} \mathrm{C} \mathrm{NMR}\left(\mathrm{CDCl}_{3}, 75 \mathrm{MHz}\right)$ : Signals for phenyl groups have not been assigned, $\delta$ 170.3 (C, C-1'’), 170.1 (C, C-1), 168.5 (C, C-1'), 148.8 (C, C-6'’), 147.7 (C, C-3a), 147.2 (C, C-3a'’), 146.0 (C, C-7'’), 145.1 (C, C-5), 141.8 (C, C-7), 135.6 (C, C-6), 134.6 (C, C4), 134.5 (C, C-4'’), 132.1 (C, C-5'’), 122.3 (C, C-7a' '), 120.5 (C, C-7a), 68.3 (2 × CH 2 , C3, C-3'”), $61.9\left(\mathrm{CH}_{2}, \mathrm{C}-3\right.$ ') $24.6\left(\mathrm{CH}_{2}, \mathrm{C} 5-\mathrm{Et}\right), 22.6\left(\mathrm{CH}_{2}, \mathrm{C} 7-\mathrm{Et}\right), 20.9\left(\mathrm{CH}_{2}, \mathrm{C} 7{ }^{\prime}\right.$-Et $)$, $15.9\left(\mathrm{CH}_{3}, \mathrm{C} 7\right.$ ' '-Et), $15.6\left(\mathrm{CH}_{3}, \mathrm{C} 7-\mathrm{Et}\right), 15.5\left(\mathrm{CH}_{3}, \mathrm{C} 5-\mathrm{Et}\right)$. HRMS $m / z$ calcd. for $\mathrm{C}_{42} \mathrm{H}_{37} \mathrm{O}_{6}$ $[\mathrm{M}+\mathrm{H}]^{+} 637.2585$, found 637.2574 .

\section{Compounds 3ae}

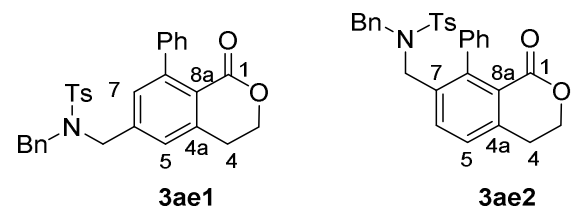

Obtained as a pale yellow liquid consisting of an inseparable mixture of 3ae1 and 3ae2 in an approx. ratio $3: 1(0.199 \mathrm{~g}, 0.40 \mathrm{mmol},<40 \%$ yield, contaminated with inseparable unidentified side-products). IR (film) $\left(\mathrm{cm}^{-1}\right): 3028,2920,2903,1728,1339$, 1159, 1094. ${ }^{1} \mathrm{H}$ NMR $\left(\mathrm{CDCl}_{3}, 300 \mathrm{MHz}\right)$ : Major isomer 3ae1: $\delta$ 7.78-7.71 (m, 2H, Ts), 7.46-7.02 (m, 12H, Ar-H, Ts), 6.96 (s, 1H, 5-H), 6.83 (s, 1H, 7-H), 4.44 (t, J= 5.8, 2H, 3H), 4.34 (s, 2H, Bn), $4.33\left(\mathrm{~s}, 2 \mathrm{H}, 6-\mathrm{CH}_{2}\right), 2.90(\mathrm{t}, J=5.7,2 \mathrm{H}, 4-\mathrm{H}), 2.43$ (s, 3H, Ts). Minor isomer 3ae2: $\delta 7.69(\mathrm{~d}, J=8.1 \mathrm{~Hz}, 1 \mathrm{H}, 6-\mathrm{H}), 7.59-7.52(\mathrm{~m}, 2 \mathrm{H}, \mathrm{Ts}), 7.46-6.78(\mathrm{~m}, 13 \mathrm{H}$, Ar-H, Ts, 5-H), $4.41(\mathrm{t}, J=5.7,2 \mathrm{H}, 3-\mathrm{H}), 4.11(\mathrm{~s}, 2 \mathrm{H}, \mathrm{Bn}), 4.03\left(\mathrm{~s}, 2 \mathrm{H}, 7-\mathrm{CH}_{2}\right), 3.01(\mathrm{t}, J=$ 5.7, 2H, 4-H), 2.40 (s, 3H, Ts). $\mathrm{RMN} \mathrm{de}{ }^{13} \mathrm{C}\left(\mathrm{CDCl}_{3}, 300 \mathrm{MHz}\right)$ : Major isomer 3ae1: $\delta$ 
163.1 (C, C-1), 145.7 (C, C-8), 143.5 (C, Ts), 141.1 (C, C-4a), 140.7 (C, C-6), 140.6 (C, Ar), 136.8 (C, Ts), 135.3 (C, Bn), $130.3(\mathrm{CH}, \mathrm{C}-7), 129.7(2 \times \mathrm{CH}, \mathrm{Ar}), 128.6(2 \times \mathrm{CH}$, Ar $), 128.2(2 \times \mathrm{CH}, \mathrm{Ar}), 128.1(2 \times \mathrm{CH}, \mathrm{Ar}), 127.8(2 \times \mathrm{CH}, \mathrm{Ar}), 127.0(2 \times \mathrm{CH}, \mathrm{Ar})$ $126.0(\mathrm{CH}, \mathrm{C}-5), 122.5$ (C, C-8a), $66.5\left(\mathrm{CH}_{2}, \mathrm{C}-3\right), 52.0\left(\mathrm{CH}_{2}, \mathrm{Bn}\right), 50.8\left(\mathrm{CH}_{2}, 6-\mathrm{CH}_{2}\right)$, $29.0\left(\mathrm{CH}_{2}, \mathrm{C}-4\right), 21.4\left(\mathrm{CH}_{3}, \mathrm{Ts}\right)$. Minor isomer 3ae2: $\delta 162.9(\mathrm{C}, \mathrm{C}-1), 143.32(\mathrm{C}, \mathrm{Ts})$, 143.28 (C, C-8), 139.5 (C, C-4a), 138.4 (C, Ar), 136.5 (C, Ar), 135.3 (C, C-7) 135.1 (C, Bn), $132.9(2 \times \mathrm{CH}, \mathrm{Ts}), 132.7(\mathrm{CH}, \mathrm{C}-6), 129.5(2 \times \mathrm{CH}, \mathrm{Ar}), 128.5(2 \times \mathrm{CH}, \mathrm{Ar}), 128.1$ $(2 \times \mathrm{CH}, \mathrm{Ar}), 127.9(2 \times \mathrm{CH}, \mathrm{Ar}), 127.6(\mathrm{CH}, \mathrm{Ar}), 127.0(2 \times \mathrm{CH}, \mathrm{Ts}), 126.5(\mathrm{CH}, \mathrm{C}-5)$, 123.9 (C, C-8a), $66.5\left(\mathrm{CH}_{2}, \mathrm{C}-3\right), 52.2\left(\mathrm{CH}_{2}, \mathrm{Bn}\right), 48.7\left(\mathrm{CH}_{2}, \mathrm{C} 7-\mathrm{CH}_{2}\right), 28.9\left(\mathrm{CH}_{2}, \mathrm{C}-4\right)$, $21.4\left(\mathrm{CH}_{3}, \mathrm{Ts}\right)$. HRMS $m / z$ calcd. for $\mathrm{C}_{30} \mathrm{H}_{28} \mathrm{NO}_{4} \mathrm{~S}[\mathrm{M}+\mathrm{H}]^{+} 498.1734$, found 498.1731 .

\section{Preparation of resin $1 b$}

To $1.0 \mathrm{~g}$ of BOBA resin prepared according to Kobayashi and Aoki (7, approximate loading: $1.00 \mathrm{mmol} / \mathrm{g})$ suspended in dichloromethane $(9 \mathrm{~mL})$ was added p-toluenesulfonyl chloride $(0.593 \mathrm{~g}, 3.10 \mathrm{mmol})$ and triethylamine $(0.65 \mathrm{~mL}, 1.57 \mathrm{mmol})$. The reaction mixture was stirred at room temperature for 18 hours and then filtered. The remaining resin was then washed with dichloromethane $(7 \times 10 \mathrm{~mL})$ and dried under reduced pressure. To this resin in DMF $(18 \mathrm{~mL})$ was added cesium carbonate $(1.77 \mathrm{~g}, 5.4 \mathrm{mmol})$ and propargyl bromide ( $80 \%$ in toluene, $1.2 \mathrm{~mL}, 10.8 \mathrm{mmol})$. The reaction mixture was stirred at room temperature for 24 hours and then filtered. The remaining solid was washed with DMF $(2 \times$ $10 \mathrm{~mL}), \mathrm{DMF}: \mathrm{H}_{2} \mathrm{O}(2 \times 10 \mathrm{~mL}), \mathrm{H}_{2} \mathrm{O}(2 \times 10 \mathrm{~mL}), \mathrm{DMF}(3 \times 10 \mathrm{~mL}), \mathrm{DCM}$ and $\mathrm{MeOH}$ (alternated $5 \times 10 \mathrm{~mL}$ each) and DCM $(3 \times 10 \mathrm{~mL})$. The final resin was dried under 
reduced pressure to afford resin $\mathbf{1 b}$ (approx. $1.18 \mathrm{~g}$ ). The loading of resin $\mathbf{1 b}$ was calculated based on the amount of compound 8 released upon treatment with TFA in DCM $(1 \mathrm{~mL}$ TFA/3 mL DCM for $100 \mathrm{mg}$ of resin $\mathbf{1 b}, 18 \mathrm{~h}$, room temperature). The crude mixture thus obtained was purified by flash column chromatography on silica gel (eluent hexanes/ethyl acetate) to afford known compound $\mathbf{8}$ (initial loading resin 6: 1.16 $\mathrm{mmol} / \mathrm{g}$; theoretical loading $\mathbf{1 b}: 0.88 \mathrm{mmol} / \mathrm{g}$, isolated amount 8: $13.8 \mathrm{mg}, 0.066$ mmol, calculated loading $\mathbf{1 b}: 0.66 \mathrm{mmol} / \mathrm{g}, 75 \%$ yield after five steps from resin $\mathbf{6}$ ).

\section{Compound $8^{32}$}

Colourless solid. M.p.: 74.0-75.0 ${ }^{\circ} \mathrm{C} .{ }^{1} \mathrm{H}$ NMR $\left(\mathrm{CDCl}_{3}, 300 \mathrm{MHz}\right): \delta$ 7.83-7.73 (m, 2H), 7.36-7.28 (m, 2H), $4.76(\mathrm{t}, J=5.5 \mathrm{~Hz}, 1 \mathrm{H}), 3.83(\mathrm{dd}, J=6.0 \mathrm{~Hz}, J=2.5 \mathrm{~Hz}, 2 \mathrm{H}), 2.43(\mathrm{~s}$, 3H), $2.10(\mathrm{t}, J=2.5 \mathrm{~Hz}, 1 \mathrm{H}) .{ }^{13} \mathrm{C} \mathrm{NMR}\left(\mathrm{CDCl}_{3}, 75 \mathrm{MHz}\right): \delta 143.7,136.4,129.6,127.3$, $77.8,72.9,32.7,21.4$

\section{Representative procedure for the solid-phase synthesis of phthalides 9 and 4}

A mixture of resin $\mathbf{1 b}(200 \mathrm{mg}, 0.66 \mathrm{mmol} / \mathrm{g}, 0.13 \mathrm{mmol})$, diyne 2 (0.66 mmol, 5 equiv), and Wilkinson catalyst $\left[\mathrm{Rh}\left(\mathrm{PPh}_{3}\right)_{3} \mathrm{Cl}, 6 \mathrm{mg}, 0.0065 \mathrm{mmol}\right]$ in toluene $(4.5 \mathrm{~mL}, 0.03 \mathrm{M})$ was heated at reflux. After approx. 2 hours, the reaction was filtered and the filtrate was evaporated under reduced pressure. This residue was purified by flash column chromatography on silica gel (eluent hexanes/ethyl acetate) to afford the following phthalides 4 . The remaining resin was treated overnight with TFA in DCM (1 mL TFA/3 mL DCM for $100 \mathrm{mg}$ of resin, room temperature). After that time, the solvent was evaporated and the residue purified by flash column 
chromatography on silica gel (eluent hexanes/ethyl acetate or DCM/methanol) to afford the following phthalides 9.

\section{Compounds 9ba}
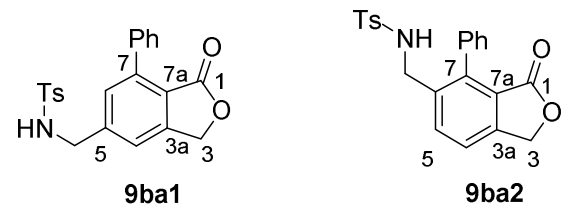

Obtained as a pale yellow liquid consisting of an inseparable mixture of $\mathbf{9 b a 1}$ and 9ba2 in an approx. ratio 2.7:1 (0.036 $\mathrm{g}, 0.09 \mathrm{mmol}, 70 \%$ yield $).$ IR (film) $\left(\mathrm{cm}^{-1}\right): 3059$, 3026, 2924, 1755, 1327, 1159. ${ }^{1} \mathrm{H}$ NMR $\left(\mathrm{CDCl}_{3}, 300 \mathrm{MHz}\right)$ : Major isomer 9ba1: $\delta$ 7.767.70 (m, 2H, Ts), 7.48-7.38 (m, 5H, Ar-H), 7.35 (s, 1H, 4-H), 7.30-7.25 (m, 2H, Ts), 7.23 (s, 1H, 6-H), 5.37 (t, $J=6.5 \mathrm{~Hz}, 1 \mathrm{H}, \mathrm{N}-\mathrm{H}), 5.19$ (s, 2H, 3-H), 4.27 (d, $J=6.5 \mathrm{~Hz}, 2 \mathrm{H}, 5-$ $\left.\mathrm{CH}_{2}\right), 2.41(\mathrm{~s}, 3 \mathrm{H}, \mathrm{Ts})$. Minor isomer 9ba2: $\delta 7.80(\mathrm{~d}, J=7.9 \mathrm{~Hz}, 1 \mathrm{H}, 5-\mathrm{H}), 7.57-7.51(\mathrm{~m}$, 2H, Ts), 7.47-7.36 (m, 3H, Ar, 4-H), 7.29-7.17 (m, 3H, Ar-H), 7.14-7.09 (m, 2H, Ts), 5.24 $(\mathrm{s}, 2 \mathrm{H}, 3-\mathrm{H}), 4.71(\mathrm{t}, J=6.3 \mathrm{~Hz}, 1 \mathrm{H}, \mathrm{N}-\mathrm{H}), 4.00\left(\mathrm{~d}, J=6.3 \mathrm{~Hz}, 2 \mathrm{H}, 6-\mathrm{CH}_{2}\right), 2.41(\mathrm{~s}, 3 \mathrm{H}$, Ts). ${ }^{13} \mathrm{C} \mathrm{NMR}\left(\mathrm{CDCl}_{3}, 75 \mathrm{MHz}\right)$ : Major isomer 9ba1: $\delta 169.3(\mathrm{C}, \mathrm{C}-1), 148.5(\mathrm{C}, \mathrm{C}-3 \mathrm{a})$, 143.7 (C, Ts), 143.2 (C, C-5), 142.7 (C, C-7), 136.7 (C, Ts), 135.7 (C, Ar), 130.1 (CH, C6), $129.6(2 \times \mathrm{CH}, \mathrm{Ts}), 129.3(2 \times \mathrm{CH}, \mathrm{Ar}), 128.4(\mathrm{CH}, \mathrm{Ar}), 127.8(2 \times \mathrm{CH}, \mathrm{Ar}), 126.9(2 \times$ CH, Ts), 121.0 (C, C-7a), 119.8 (CH, C-4), $68.1\left(\mathrm{CH}_{2}, \mathrm{C}-3\right), 46.6\left(\mathrm{CH}_{2}, \mathrm{C} 5-\mathrm{CH}_{2}\right), 21.4$ $\left(\mathrm{CH}_{3}, \mathrm{Ts}\right)$. Minor isomer 9ba2: $\delta 169.1(\mathrm{C}, \mathrm{C}-1), 146.8(\mathrm{C}, \mathrm{C}-3 \mathrm{a}), 143.4(\mathrm{C}, \mathrm{Ts}), 141.1(\mathrm{C}$, C-7), 136.2 (C, Ts), 136.1 (C, C-6), 135.0 (CH, C-5), 133.8 (C, Ar), 129.5 (2 × CH, Ts), $128.6(2 \times \mathrm{CH}, \mathrm{Ar}), 128.2(2 \times \mathrm{CH}, \mathrm{Ar}), 128.1(\mathrm{CH}, \mathrm{Ar}), 126.8(2 \times \mathrm{CH}, \mathrm{Ts}), 123.2(\mathrm{C}, \mathrm{C}-$ 
7a), 121.4 (CH, C-4), $68.0\left(\mathrm{CH}_{2}, \mathrm{C}-3\right), 43.9\left(\mathrm{CH}_{2}, \mathrm{C} 6-\mathrm{CH}_{2}\right), 21.4\left(\mathrm{CH}_{3}, \mathrm{Ts}\right) . \mathrm{HRMS} m / z$ calcd. for $\mathrm{C}_{22} \mathrm{H}_{20} \mathrm{NO}_{4} \mathrm{~S}[\mathrm{M}+\mathrm{H}]^{+}$394.1108, found 394.1092.

\section{Compounds 9bb}
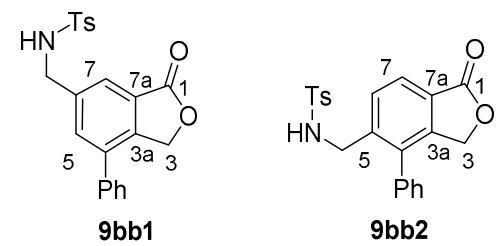

Obtained as a pale yellow liquid consisting of an inseparable mixture of $\mathbf{9 b b 1}$ and 9bb2 in an approx. ratio 1.3:1 (0.020 g, $0.052 \mathrm{mmol}, 40 \%$ yield). IR (film) $\left(\mathrm{cm}^{-1}\right)$ : 3026, 2922, 1751, 1327, 1159. ${ }^{1} \mathrm{H}$ NMR $\left(\mathrm{CDCl}_{3}, 300 \mathrm{MHz}\right)$ : Major isomer 9bb1: $\delta$ 7.79-7.72 (m, 2H, Ts), 7.66 (s, 1H, 7-H), 7.64 (s, 1H, 5-H), 7.53-7.40 (m, 3H, Ar-H), 7.40-7.34 (m, 2H, Ar-H), 7.33-7.27 (m, 2H, Ts), $5.36(\mathrm{~s}, 2 \mathrm{H}, 3-\mathrm{H}), 4.97$ (t, $J=6.5 \mathrm{~Hz}, 1 \mathrm{H}, \mathrm{N}-\mathrm{H}), 4.30(\mathrm{~d}, J=$ $\left.6.3 \mathrm{~Hz}, 2 \mathrm{H}, 6-\mathrm{CH}_{2}\right), 2.42$ (s, 3H, Ts). Minor isomer 9bb2: $\delta 7.84(\mathrm{~d}, J=8.1 \mathrm{~Hz}, 1 \mathrm{H}, 7-\mathrm{H})$, $7.60(\mathrm{~d}, J=8.0 \mathrm{~Hz}, 1 \mathrm{H}, 6-\mathrm{H}), 7.60-7.55$ (m, 2H, Ts), 7.53-7.28 (m, 3H, Ar-H), 7.24-7.18 (m, 2H, Ts), 7.17-7.11 (m, 2H, Ar-H), 4.99 (s, 2H, 3-H), 4.60 (t, J=6.2 Hz, 1H, N-H), 4.10 $\left(\mathrm{d}, J=6.3 \mathrm{~Hz}, 2 \mathrm{H}, 5-\mathrm{CH}_{2}\right), 2.42(\mathrm{~s}, 3 \mathrm{H}, \mathrm{Ts}) .{ }^{13} \mathrm{C} \mathrm{NMR}\left(\mathrm{CDCl}_{3}, 75 \mathrm{MHz}\right)$ : Major isomer 9bb1: $\delta 170.5$ (C, C-1), 143.8 (C, Ts), 143.7 (C, C-3a), 138.9 (C, C-6), 137.4 (C, C-4), 136.9 (C, Ar), 136.7 (C, Ts), 133.4 (CH, C-5), $129.7(2 \times \mathrm{CH}, \mathrm{Ts}), 129.1(2 \times \mathrm{CH}, \mathrm{Ar})$ $128.6(\mathrm{CH}, \mathrm{Ar}), 127.5(2 \times \mathrm{CH}, \mathrm{Ar}), 127.0(2 \times \mathrm{CH}, \mathrm{Ts}), 126.8(\mathrm{C}, \mathrm{C}-7 \mathrm{a}), 123.4(\mathrm{CH}, \mathrm{C}-7)$, $69.5\left(\mathrm{CH}_{2}, \mathrm{C}-3\right), 46.6\left(\mathrm{CH}_{2}, \mathrm{C} 6-\mathrm{CH}_{2}\right), 21.4\left(\mathrm{CH}_{3}\right.$, Ts $)$. Minor isomer 9bb2: $\delta 170.6(\mathrm{C}, \mathrm{C}-$ 1), 146.1 (C, C-3a), 143.6 (C, Ts), 140.4 (C, C-5), 136.4 (C, Ts), 136.2 (C, C-4), 134.8 (C, Ar), $130.3(\mathrm{CH}, \mathrm{C}-6), 129.6(2 \times \mathrm{CH}, \mathrm{Ts}), 129.2(2 \times \mathrm{CH}, \mathrm{Ar}), 128.6(\mathrm{CH}, \mathrm{Ar}), 128.1(2 \times$ 
CH, Ar), $126.9(2 \times \mathrm{CH}, \mathrm{Ts}), 125.3(\mathrm{C}, \mathrm{C}-7 \mathrm{a}), 124.9(\mathrm{CH}, \mathrm{C}-7), 69.1\left(\mathrm{CH}_{2}, \mathrm{C}-3\right), 44.3$ $\left(\mathrm{CH}_{2}, \mathrm{C} 5-\mathrm{CH}_{2}\right), 21.4\left(\mathrm{CH}_{3}\right.$, Ts). HRMS $m / z$ calcd. for $\mathrm{C}_{22} \mathrm{H}_{20} \mathrm{NO}_{4} \mathrm{~S}[\mathrm{M}+\mathrm{H}]^{+}$394.1108, found 394.1101.

\section{Compounds 9bc}

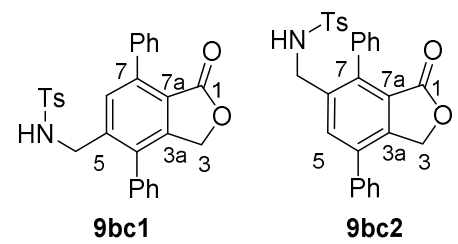

Obtained as a colorless to pale yellow solid consisting of an inseparable mixture of 9bc1 and 9bc2 in an approx. ratio 1.2:1 (0.037 g, $0.078 \mathrm{mmol}, 60 \%$ yield). M.p.: 91.8-96.8 ${ }^{\circ} \mathrm{C}$. IR (film) $\left(\mathrm{cm}^{-1}\right): 3059,3026,2920,1755,1327,1159 .{ }^{1} \mathrm{H}$ NMR $\left(\mathrm{CDCl}_{3}, 300 \mathrm{MHz}\right)$ : Major isomer 9bc1: $\delta$ 7.60-7.13 (m, 15H, Ar-H, Ts, 6-H), $4.96(\mathrm{~s}, 2 \mathrm{H}, 3-\mathrm{H}), 4.62(\mathrm{t}, J=6.4$ $\mathrm{Hz}, 1 \mathrm{H}, \mathrm{N}-\mathrm{H}), 4.14\left(\mathrm{~d}, J=6.4 \mathrm{~Hz}, 2 \mathrm{H}, 5-\mathrm{CH}_{2}\right), 2.37(\mathrm{~s}, 3 \mathrm{H}, \mathrm{Ts})$. Minor isomer 9bc2: $\delta 7.74$ (s, 1H, 5-H), 7.60-7.13 (m, 14H, Ar-H, Ts), $5.32(\mathrm{~s}, 2 \mathrm{H}, 3-\mathrm{H}), 4.51(\mathrm{t}, J=6.5 \mathrm{~Hz}, 1 \mathrm{H}, \mathrm{N}-$ H), 4.08 (d, $\left.J=6.4 \mathrm{~Hz}, 2 \mathrm{H}, 6-\mathrm{CH}_{2}\right), 2.38$ (s, 3H, Ts). ${ }^{13} \mathrm{C}$ NMR $\left(\mathrm{CDCl}_{3}, 75 \mathrm{MHz}\right)$ : Only signals attributed to the phthalide core are listed, major isomer 9bc1: $\delta 169.3(\mathrm{C}, \mathrm{C}-1)$, 147.5 (C, C-3a), 140.1 (C, C-5), 134.7 (C, C-4), 131.6 (CH, C-6), 121.2 (C, C-7a), 67.9 $\left(\mathrm{CH}_{2}, \mathrm{C}-3\right), 44.3\left(\mathrm{CH}_{2}, \mathrm{C} 5-\mathrm{CH}_{2}\right)$. Minor isomer 9bc2: $\delta 169.0(\mathrm{C}, \mathrm{C}-1), 144.3(\mathrm{C}, \mathrm{C}-3 \mathrm{a})$, 139.9 (C, C-7), 136.8 (C, C-6), 136.2 (C, C-4), 134.6 (CH, C-5), 123.9 (C, C-7a), 68.0 $\left(\mathrm{CH}_{2}, \mathrm{C}-3\right), 44.2\left(\mathrm{CH}_{2}, \mathrm{C} 6-\mathrm{CH}_{2}\right)$. HRMS $m / z$ calcd. for $\mathrm{C}_{28} \mathrm{H}_{24} \mathrm{NO}_{4} \mathrm{~S}[\mathrm{M}+\mathrm{H}]^{+}$470.1421, found 470.1417 . 


\section{Compounds 9bd}
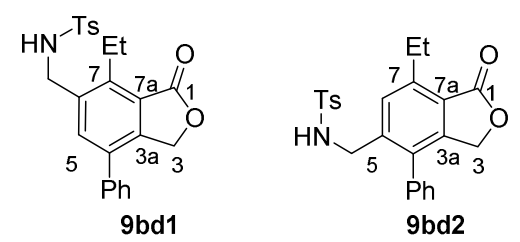

Obtained as a pale yellow liquid consisting of an inseparable mixture of $\mathbf{9 b d 1}$ and 9bd2 in an approx. ratio 1.2:1 (0.033 g, $0.078 \mathrm{mmol}, 60 \%$ yield). IR (film) $\left(\mathrm{cm}^{-1}\right): 3026$, 2968, 2930, 2874, 1747, 1329, 1159. ${ }^{1} \mathrm{H}$ NMR $\left(\mathrm{CDCl}_{3}, 300 \mathrm{MHz}\right)$ : Major isomer 9bd1: $\delta$ 7.78-7.72 (m, 2H, Ts), $7.52(\mathrm{~s}, 1 \mathrm{H}, 5-\mathrm{H}), 7.49-7.36(\mathrm{~m}, 3 \mathrm{H}, \mathrm{Ar}-\mathrm{H})$, 7.34-7.24 (m, 4H, Ts, Ar-H), $5.24(\mathrm{~s}, 2 \mathrm{H}, 3-\mathrm{H}), 5.13(\mathrm{t}, J=6.0 \mathrm{~Hz}, 1 \mathrm{H}, \mathrm{N}-\mathrm{H}), 4.27\left(\mathrm{~d}, J=6.1 \mathrm{~Hz}, 2 \mathrm{H}, 6-\mathrm{CH}_{2}\right)$, 3.07 (q, $J=7.5 \mathrm{~Hz}, 2 \mathrm{H}, 7-\mathrm{Et}), 2.40$ (s, 3H, Ts), 1.14 (t, $J=7.5 \mathrm{~Hz}, 3 \mathrm{H}, 7-\mathrm{Et})$. Minor isomer 9bd2: $\delta$ 7.60-7.54 (m, 2H, Ts), 7.49-7.35 (m, 3H, Ar-H), 7.34-7.24 (overlapping signal, 1H, 6-H), 7.23-7.16 (m, 2H, Ts), 7.14-7.07 (m, 2H, Ar-H), 4.89 (s, 2H, 3-H), 4.88 (t, $J=6.3$ Hz, 1H, N-H), 4.05 (d, $\left.J=6.3 \mathrm{~Hz}, 2 \mathrm{H}, 5-\mathrm{CH}_{2}\right), 3.05$ (q, $\left.J=7.6 \mathrm{~Hz}, 2 \mathrm{H}, 7-\mathrm{Et}\right), 2.40$ (s, 3H, Ts), 1.24 (t, $J=7.6 \mathrm{~Hz}, 3 \mathrm{H}, 7-\mathrm{Et}) .{ }^{13} \mathrm{C} \mathrm{NMR}\left(\mathrm{CDCl}_{3}, 75 \mathrm{MHz}\right)$ : Major isomer 9bd1: $\delta$ 170.3 (C, C-1), 144.6 (C, C-3a), 143.6 (C, Ts), 143.3 (C, C-7), 137.1 (C, Ar), 136.5 (C, Ar), 135.7 (C, C-6), 134.7 (CH, C-5), 134.3 (C, C-4), $129.6(2 \times \mathrm{CH}, \mathrm{Ts}), 128.9(2 \times \mathrm{CH}, \mathrm{Ar})$, $128.2(\mathrm{CH}, \mathrm{Ar}), 127.5(2 \times \mathrm{CH}, \mathrm{Ar}), 127.0(2 \times \mathrm{CH}, \mathrm{Ts}), 123.5(\mathrm{C}, \mathrm{C}-7 \mathrm{a}), 68.1\left(\mathrm{CH}_{2}, \mathrm{C}-3\right)$, $43.4\left(\mathrm{CH}_{2}, \mathrm{C} 6-\mathrm{CH}_{2}\right), 21.4\left(\mathrm{CH}_{3}, \mathrm{Ts}\right), 19.5\left(\mathrm{CH}_{2}, \mathrm{C} 7-\mathrm{Et}\right), 15.2\left(\mathrm{CH}_{3}, \mathrm{C} 7-\mathrm{Et}\right)$. Minor isomer 9bd2: $\delta 170.5$ (C, C-1), 146.7 (C, C-3a), 145.2 (C, C-7), 143.5 (C, Ts), 140.0 (C, C-5), 136.5 (C, Ts), 134.8 (C, Ar), 133.4 (C, C-4), 129.9 (CH, C-6), 129.6 (2 × CH, Ts), 129.1 (2 $\times \mathrm{CH}, \mathrm{Ar}), 128.3(\mathrm{CH}, \mathrm{Ar}), 128.2(2 \times \mathrm{CH}, \mathrm{Ar}), 126.8(2 \times \mathrm{CH}, \mathrm{Ts}), 122.0(\mathrm{C}, \mathrm{C}-7 \mathrm{a}), 68.3$ 
$\left(\mathrm{CH}_{2}, \mathrm{C}-3\right), 44.2\left(\mathrm{CH}_{2}, \mathrm{C} 5-\mathrm{CH}_{2}\right), 23.8\left(\mathrm{CH}_{2}, \mathrm{C} 7-\mathrm{Et}\right), 21.3\left(\mathrm{CH}_{3}, \mathrm{Ts}\right), 14.8\left(\mathrm{CH}_{3}, \mathrm{C} 7-\mathrm{Et}\right)$. HRMS $m / z$ calcd. for $\mathrm{C}_{24} \mathrm{H}_{24} \mathrm{NO}_{4} \mathrm{~S}[\mathrm{M}+\mathrm{H}]^{+} 422.1421$, found 422.1433 .

\section{Compounds 9bg}
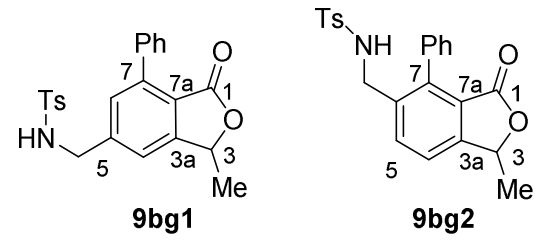

Obtained as a pale yellow solid consisting of an inseparable mixture of 9bg1 and 9bg2 in an approx. ratio 2.7:1 (0.037 g, $0.091 \mathrm{mmol}, 70 \%$ yield). M.p.: $174.9-178.9^{\circ} \mathrm{C}$. IR (film) $\left(\mathrm{cm}^{-1}\right): 3024,2980,2926,1751,1329,1159,1051 .{ }^{1} \mathrm{H}$ NMR $\left(\mathrm{CDCl}_{3}, 300 \mathrm{MHz}\right)$ : Major isomer 9bg1: $\delta$ 7.75-7.69 (m, 2H, Ts), 7.47-7.33 (m, 5H, Ar-H), 7.30-7.23 (m, 2H, Ts), $7.28(\mathrm{~s}, 1 \mathrm{H}, 4-\mathrm{H}), 7.21(\mathrm{~s}, 1 \mathrm{H}, 6-\mathrm{H}), 5.42(\mathrm{q}, J=6.5 \mathrm{~Hz}, 1 \mathrm{H}, 3-\mathrm{H}), 5.22(\mathrm{t}, J=5.6 \mathrm{~Hz}$, 1H, N-H), 4.28 (d, $\left.J=6.1 \mathrm{~Hz}, 2 \mathrm{H}, 5-\mathrm{CH}_{2}\right), 2.40$ (s, 3H, Ts), 1.58 (d, J=6.6 Hz, 3H, 3$\mathrm{CH}_{3}$ ). Minor isomer 9bg2: $\delta 7.77(\mathrm{~d}, J=7.9 \mathrm{~Hz}, 1 \mathrm{H}, 5-\mathrm{H}), 7.55-7.49(\mathrm{~m}, 2 \mathrm{H}, \mathrm{Ts}), 7.47-7.33$ (m, 4H, Ar-H, 4-H), 7.22-7.16 (m, 2H, Ts), 7.14-7.06 (m, 2H, Ar-H), 5.47 (q, $J=6.5 \mathrm{~Hz}$ 1H, 3-H), 4.59 (t, $J=6.5 \mathrm{~Hz}, 1 \mathrm{H}, \mathrm{N}-\mathrm{H}), 4.00\left(\mathrm{~d}, J=6.3 \mathrm{~Hz}, 2 \mathrm{H}, 6-\mathrm{CH}_{2}\right), 2.40(\mathrm{~s}, 3 \mathrm{H}, \mathrm{Ts})$, $1.62\left(\mathrm{~d}, J=6.7 \mathrm{~Hz}, 3 \mathrm{H}, 3-\mathrm{CH}_{3}\right) .{ }^{13} \mathrm{C} \mathrm{NMR}\left(\mathrm{CDCl}_{3}, 75 \mathrm{MHz}\right)$ : Major isomer 9bg1: $\delta 168.6$ (C, C-1), 153.1 (C, C-3a), 143.7 (C, Ts), 143.2 (C, C-5), 142.8 (C, C-7), 136.7 (C, Ts), 135.8 (C, Ar), $130.1(\mathrm{CH}, \mathrm{C}-6), 129.7(2 \times \mathrm{CH}, \mathrm{Ts}), 129.3(2 \times \mathrm{CH}, \mathrm{Ar}), 128.4(\mathrm{CH}, \mathrm{Ar})$ $127.8(2 \times \mathrm{CH}, \mathrm{Ar}), 127.0(2 \times \mathrm{CH}, \mathrm{Ts}), 121.1(\mathrm{C}, \mathrm{C}-7 \mathrm{a}), 119.3(\mathrm{CH}, \mathrm{C}-4), 75.9(\mathrm{CH}, \mathrm{C}-3)$, $46.7\left(\mathrm{CH}_{2}, \mathrm{C} 5-\mathrm{CH}_{2}\right), 21.4\left(\mathrm{CH}_{3}, \mathrm{Ts}\right), 20.3\left(\mathrm{CH}_{3}, \mathrm{C} 3-\mathrm{CH}_{3}\right)$. Minor isomer 9bg2: $\delta 168.4(\mathrm{C}$, 
C-1), 151.4 (C, C-3a), 143.4 (C, Ts), 141.0 (C, C-7), 136.3 (C, Ts), 136.0 (C, C-6), 135.1 (CH, C-5), $133.9(\mathrm{C}, \mathrm{Ar}), 129.5(2 \times \mathrm{CH}, \mathrm{Ts}), 128.7(\mathrm{CH}, \mathrm{Ar}), 128.5(2 \times \mathrm{CH}, \mathrm{Ar}), 128.2(2$ $\times \mathrm{CH}, \mathrm{Ar}), 126.8(2 \times \mathrm{CH}, \mathrm{Ts}), 123.2(\mathrm{C}, \mathrm{C}-7 \mathrm{a}), 120.9(\mathrm{CH}, \mathrm{C}-4), 75.8(\mathrm{CH}, \mathrm{C}-3), 44.0$ $\left(\mathrm{CH}_{2}, \mathrm{C} 6-\mathrm{CH}_{2}\right), 21.4\left(\mathrm{CH}_{3}, \mathrm{Ts}\right), 20.3\left(\mathrm{CH}_{3}, \mathrm{C} 3-\mathrm{CH}_{3}\right)$. HRMS $m / z$ calcd. for $\mathrm{C}_{23} \mathrm{H}_{22} \mathrm{NO}_{4} \mathrm{~S}[\mathrm{M}$ $+\mathrm{H}]^{+}$408.1264, found 408.1269 .

\section{Compounds 4g}

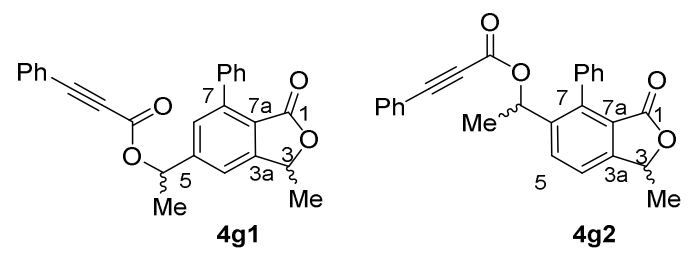

Obtained as a pale yellow liquid consisting of an inseparable mixture of $4 \mathbf{g} 1$ and 4g2 in an approx. ratio 3.3:1 (0.068 g, $0.171 \mathrm{mmol}, 52 \%$ yield). IR (film) $\left(\mathrm{cm}^{-1}\right): 3022$, 2982, 2932, 2212, 1761, 1707, 1283, 1188, 1171. ${ }^{1} \mathrm{H}$ NMR ( $\left.\mathrm{CDCl}_{3}, 300 \mathrm{MHz}\right): \delta 7.910$ (d, $\left.J=8.1 \mathrm{~Hz}, 1 \mathrm{H}, 5-\mathrm{H}^{*}\right), 7.909\left(\mathrm{~d}, J=8.0 \mathrm{~Hz}, 1 \mathrm{H}, 5-\mathrm{H}^{*}\right), 7.64-7.10$ (overlapping signals, $46 \mathrm{H}, \operatorname{Ar}-\mathrm{H}$ ), $6.11(\mathrm{q}, J=6.7 \mathrm{~Hz}, 2 \mathrm{H}), 5.93-5.81$ (overlapping signals, $2 \mathrm{H}$ ), 5.61-5.43 (overlapping signals, 4H), 1.73-1.60 (overlapping signals, 18H), 1.51-1.43 (overlapping signals, $6 \mathrm{H}) .{ }^{13} \mathrm{C} \mathrm{NMR}\left(\mathrm{CDCl}_{3}, 75 \mathrm{MHz}\right): \delta 168.6,168.4,153.11,153.09,153.02,152.98$, $152.73,152.72,151.3,147.3,147.1,143.01,142.99,141.1,139.93,139.90,136.0,133.9$, $132.9,132.8,131.1,130.7,130.6,129.4,129.0,128.8,128.64,128.62,128.5,128.4,128.3$ $128.2,127.9,127.8,123.1,121.6,121.5,121.2,119.3,119.2,117.9,117.6,87.1,86.4,80.4$ $80.3,76.0,75.7,73.40,73.36,70.83,70.79,22.3,22.2,22.1,20.4,20.32,20.27$. HRMS $m / z$ calcd. for $\mathrm{C}_{26} \mathrm{H}_{20} \mathrm{NaO}_{4}[\mathrm{M}+\mathrm{Na}]^{+} 419.1254$, found 419.1252 . 


\section{Compounds 9bh}
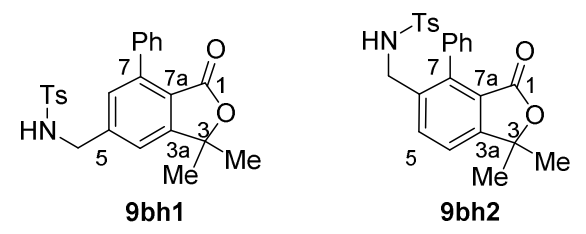

Obtained as a pale yellow liquid consisting of an inseparable mixture of $9 \mathbf{b h} \mathbf{1}$ and 9bh2 in an approx. ratio 2.5:1 (0.033 g, $0.078 \mathrm{mmol}, 60 \%$ yield). IR (film) $\left(\mathrm{cm}^{-1}\right): 3026$, 2978, 2928, 1751, 1329, 1159, 1092. ${ }^{1} \mathrm{H}$ NMR $\left(\mathrm{CDCl}_{3}, 300 \mathrm{MHz}\right)$ : Major isomer 9bh1: $\delta$ 7.76-7.71 (m, 2H, Ts), 7.49-7.30 (m, 5H, Ar-H), 7.30-7.25 (m, 2H, Ts), 7.24 (s, 1H, 4-H), 7.19 (s, 1H, 6-H), 5.16 (t, $J=6.5 \mathrm{~Hz}, 1 \mathrm{H}, \mathrm{N}-\mathrm{H}), 4.29$ (d, $\left.J=6.5 \mathrm{~Hz}, 2 \mathrm{H}, 5-\mathrm{CH}_{2}\right), 2.40$ (s, 3H, Ts), 1.61 (s, 6H, 3- $\left.\mathrm{CH}_{3}\right)$. Minor isomer 9bh2: $\delta 7.76(\mathrm{~d}, J=8.0 \mathrm{~Hz}, 1 \mathrm{H}, 5-\mathrm{H}), 7.55-$ $7.48(\mathrm{~m}, 2 \mathrm{H}, \mathrm{Ts}), 7.48-7.30(\mathrm{~m}, 3 \mathrm{H}, \mathrm{Ar}-\mathrm{H}), 7.33(\mathrm{~d}, J=8.0 \mathrm{~Hz}, 1 \mathrm{H}, 4-\mathrm{H}), 7.22-7.16(\mathrm{~m}, 2 \mathrm{H}$, Ts), 7.14-7.09 (m, 2H, Ar-H), 4.53 (t, $J=6.2 \mathrm{~Hz}, 1 \mathrm{H}, \mathrm{N}-\mathrm{H}), 4.01$ (d, $J=6.4 \mathrm{~Hz}, 2 \mathrm{H}, 6-$ $\left.\mathrm{CH}_{2}\right), 2.41$ (s, 3H, Ts), $1.64\left(\mathrm{~s}, 6 \mathrm{H}, 3-\mathrm{CH}_{3}\right) .{ }^{13} \mathrm{C} \mathrm{NMR}\left(\mathrm{CDCl}_{3}, 75 \mathrm{MHz}\right)$ : Major isomer 9bh1: $\delta 167.9$ (C, C-1), 157.0 (C, C-3a), 143.8 (C, Ts), 143.1 (C, C-5), 142.9 (C, C-7), 136.7 (C, Ts), 135.9 (C, Ar), $129.9(\mathrm{CH}, \mathrm{C}-6), 129.7(2 \times \mathrm{CH}, \mathrm{Ts}), 129.3(2 \times \mathrm{CH}, \mathrm{Ar})$, $128.2(\mathrm{CH}, \mathrm{Ar}), 127.8(2 \times \mathrm{CH}, \mathrm{Ar}), 127.0(2 \times \mathrm{CH}, \mathrm{Ts}), 120.6(\mathrm{C}, \mathrm{C}-7 \mathrm{a}), 118.4(\mathrm{CH}, \mathrm{C}-4)$, $83.3(\mathrm{C}, \mathrm{C}-3), 46.7\left(\mathrm{CH}_{2}, \mathrm{C} 5-\mathrm{CH}_{2}\right), 27.4\left(2 \times \mathrm{CH}_{3}, \mathrm{C} 3-\mathrm{CH}_{3}\right), 21.4\left(\mathrm{CH}_{3}, \mathrm{Ts}\right)$. Minor isomer 9bh2: $\delta 167.8$ (C, C-1), 155.3 (C, C-3a), 143.4 (C, Ts), 141.1 (C, C-7), 136.4 (C, Ts), 135.8 (C, C-6), 135.1 (CH, C-5), 134.0 (C, Ar), 129.5 (2 × CH, Ts), 129.4 (2× CH, Ar), $128.5(2$ $\times \mathrm{CH}, \mathrm{Ar}), 128.2(\mathrm{CH}, \mathrm{Ar}), 126.8(2 \times \mathrm{CH}, \mathrm{Ts}), 122.7(\mathrm{C}, \mathrm{C}-7 \mathrm{a}), 120.0(\mathrm{CH}, \mathrm{C}-4), 83.2(\mathrm{C}$, 


\section{C-3), $44.1\left(\mathrm{CH}_{2}, \mathrm{C} 6-\mathrm{CH}_{2}\right), 27.3\left(2 \times \mathrm{CH}_{3}, \mathrm{C} 3-\mathrm{CH}_{3}\right), 21.4\left(\mathrm{CH}_{3}, \mathrm{Ts}\right)$. HRMS $m / z$ calcd. for $\mathrm{C}_{24} \mathrm{H}_{24} \mathrm{NO}_{4} \mathrm{~S}[\mathrm{M}+\mathrm{H}]^{+}$422.1421, found 422.1404 .}

\section{Compounds 9bi}
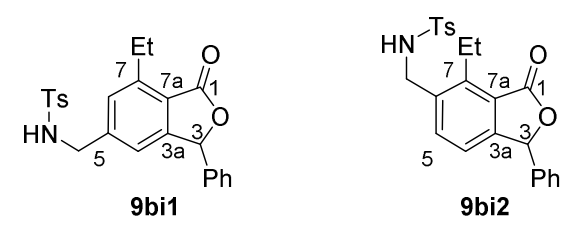

Obtained as a pale yellow liquid consisting of an inseparable mixture of $\mathbf{9 b i 1}$ and 9bi2 in an approx. ratio 2.8:1 $\left(0.030 \mathrm{~g}, 0.071 \mathrm{mmol}, 55 \%\right.$ yield). IR (film) $\left(\mathrm{cm}^{-1}\right): 3030$, 2970, 2928, 2872, 1747, 1329, 1159. ${ }^{1} \mathrm{H} \mathrm{NMR}\left(\mathrm{CDCl}_{3}, 300 \mathrm{MHz}\right)$ : Major isomer 9bi1: $\delta$ 7.72-7.65 (m, 2H, Ts), 7.39-7.33 (m, 3H, Ar-H), 7.27-7.19 (m, 4H, Ar-H, Ts), 7.12 (s, 1H, 6-H), 6.98 (s, 1H, 4-H), $6.21(\mathrm{~s}, 1 \mathrm{H}, 3-\mathrm{H}), 4.97$ (t, $J=6.5 \mathrm{~Hz}, 1 \mathrm{H}, \mathrm{N}-\mathrm{H}), 4.16$ (d, $J=6.5$ $\mathrm{Hz}, 2 \mathrm{H}, 5-\mathrm{CH}_{2}$ ), 3.17-2.97 (m, 2H, 7-Et), 2.41 (s, 3H, Ts), 1.24 (t, J=7.5 Hz, 3H, 7-Et). Minor isomer 9bi2: $\delta$ 7.78-7.72 (m, 2H, Ts), $7.50(\mathrm{~d}, J=8.0 \mathrm{~Hz}, 1 \mathrm{H}, 5-\mathrm{H}), 7.32-7.27(\mathrm{~m}$, 2H, Ts), 7.25-7.20 (m, 5H, Ar-H), 7.05 (d, J=7.9 Hz, 1H, 4-H), 6.25 (s, 1H, 3-H), 4.79 (t, $J=6.0 \mathrm{~Hz}, 1 \mathrm{H}, \mathrm{N}-\mathrm{H}), 4.22\left(\mathrm{~d}, J=6.1 \mathrm{~Hz}, 2 \mathrm{H}, 6-\mathrm{CH}_{2}\right), 3.12-2.97$ (q, $\left.J=7.4 \mathrm{~Hz}, 2 \mathrm{H}, 7-\mathrm{Et}\right)$, $2.43(\mathrm{~s}, 3 \mathrm{H}, \mathrm{Ts}), 1.16(\mathrm{t}, J=7.5 \mathrm{~Hz}, 3 \mathrm{H}, 7-\mathrm{Et}) .{ }^{13} \mathrm{C} \mathrm{NMR}\left(\mathrm{CDCl}_{3}, 75 \mathrm{MHz}\right)$ : Major isomer 9bi1: $\delta 169.7$ (C, C-1), 151.0 (C, C-3a), 146.2 (C, C-7), 143.7 (C, Ts), 143.4 (C, C-5), $136.6(\mathrm{C}, \mathrm{Ts}), 136.4(\mathrm{C}, \mathrm{Ar}), 129.6(2 \times \mathrm{CH}, \mathrm{Ts}), 128.8(2 \times \mathrm{CH}, \mathrm{Ar}), 128.6(\mathrm{CH}, \mathrm{C}-6)$, $127.0(2 \times \mathrm{CH}, \mathrm{Ts}), 126.7(2 \times \mathrm{CH}, \mathrm{Ar}), 121.7(\mathrm{C}, \mathrm{C}-7 \mathrm{a}), 119.1(\mathrm{CH}, \mathrm{C}-4), 81.5(\mathrm{CH}, \mathrm{C}-3)$ $46.8\left(\mathrm{CH}_{2}, \mathrm{C} 5-\mathrm{CH}_{2}\right), 24.0\left(\mathrm{CH}_{2}, \mathrm{C} 7-\mathrm{Et}\right), 21.4\left(\mathrm{CH}_{3}, \mathrm{Ts}\right), 14.8\left(\mathrm{CH}_{3}, \mathrm{C} 7-\mathrm{Et}\right)$. Minor isomer 9bi2: $\delta 169.7(\mathrm{C}, \mathrm{C}-1), 150.4(\mathrm{C}, \mathrm{C}-3 \mathrm{a}), 144.5(\mathrm{C}, \mathrm{C}-7), 143.7(\mathrm{C}, \mathrm{Ts}), 136.6(2 \times \mathrm{C}, \mathrm{Ar}$ 
Ts), 135.3 (C, CH, C-6, C-5), $129.7(2 \times \mathrm{CH}, \mathrm{Ts}), 129.1(2 \times \mathrm{CH}, \mathrm{Ar}), 127.0(2 \times \mathrm{CH}, \mathrm{Ts})$, $126.8(2 \times \mathrm{CH}, \mathrm{Ar}), 122.8(\mathrm{C}, \mathrm{C}-7 \mathrm{a}), 120.4(\mathrm{CH}, \mathrm{C}-4), 81.1(\mathrm{CH}, \mathrm{C}-3), 43.4\left(\mathrm{CH}_{2}, \mathrm{C} 6-\right.$ $\left.\mathrm{CH}_{2}\right), 21.4\left(\mathrm{CH}_{3}, \mathrm{Ts}\right), 19.9\left(\mathrm{CH}_{2}, \mathrm{C} 7-\mathrm{Et}\right), 15.3\left(\mathrm{CH}_{3}, \mathrm{C} 7-\mathrm{Et}\right)$. HRMS $m / z$ calcd. for $\mathrm{C}_{24} \mathrm{H}_{24} \mathrm{NO}_{4} \mathrm{~S}[\mathrm{M}+\mathrm{H}]^{+}$422.1421, found 422.1403 .

\section{Compounds $4 \mathbf{i}$}

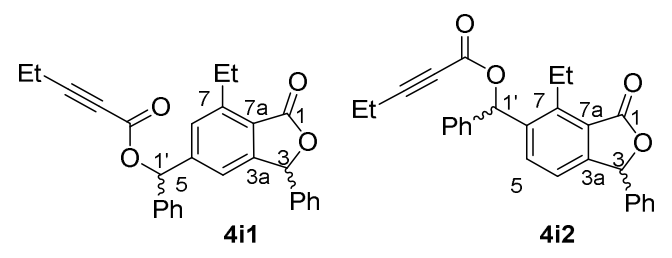

Obtained as a pale yellow liquid consisting of an inseparable mixture of $\mathbf{4 i 1}$ and $\mathbf{4 i 2}$ in an approx. ratio $2.3: 1(0.038 \mathrm{~g}, 0.089 \mathrm{mmol}, 27 \%$ yield $)$. IR (film) $\left(\mathrm{cm}^{-1}\right): 3032,2978$, 2938, 2876, 2235, 1759, 1713, 1454, 1242. ${ }^{1} \mathrm{H}$ NMR $\left(\mathrm{CDCl}_{3}, 300 \mathrm{MHz}\right): \delta 7.71(\mathrm{~d}, J=8.0$ $\mathrm{Hz}, 1 \mathrm{H}, 5-\mathrm{H}), 7.69$ (d, $J=8.0 \mathrm{~Hz}, 1 \mathrm{H}, 5-\mathrm{H}), 7.45-7.22$ (overlapping signals, 42H, Ar-H), 7.18 (s, 1H, 4-H), 7.17 (d, J=8.0 Hz, 2H, 4-H, 4-H), 7.10 (s, 1H, 4-H), 6.90 (s, 2H, 1'-H, 1'-H), 6.89 (s, 2H, 1'-H, 1'-H), 6.31 (s, 2H, 3-H, 3-H), 6.29 (s, 2H, 3-H, 3-H), 3.41-2.99 (overlapping signals, $8 \mathrm{H}, 7-\mathrm{Et}$ ), 2.42-2.28 (overlapping signals, 8H, 6'-H), 1.35-1.14 (overlapping signals, $\left.24 \mathrm{H}, 7-\mathrm{Et}, 7^{\prime}-\mathrm{H}\right) .{ }^{13} \mathrm{C} \mathrm{NMR}\left(\mathrm{CDCl}_{3}, 75 \mathrm{MHz}\right): \delta 169.6,152.6,152.50$, $152.45,150.7,150.50,150.47,146.3,146.2,146.1,146.0,144.05,143.97,138.42,138.40$ $138.37,138.2,136.41,136.37,136.34,133.69,133.67,129.4,129.13,129.07,129.0,128.8$, $128.65,128.60,128.55,128.52,128.4,128.30,128.28,128.0,127.4,127.3,127.2,127.02$ $126.96,126.9,126.8,126.7,122.8,122.2,122.1,120.42,120.38,120.3,118.8,118.3,92.1$, 92.0, 91.9, 81.6, 81.3, 81.1, 81.0, 77.5, 77.4, 74.0, 73.8, 72.05, 71.98, 66.5, 24.2, 24.1, 20.3, 
14.85, 14.81, 12.34, 12.26. HRMS $m / z$ calcd. for $\mathrm{C}_{28} \mathrm{H}_{25} \mathrm{O}_{4}[\mathrm{M}+\mathrm{H}]^{+}$425.1747, found 425.1745 .

\section{Compounds 9bj}
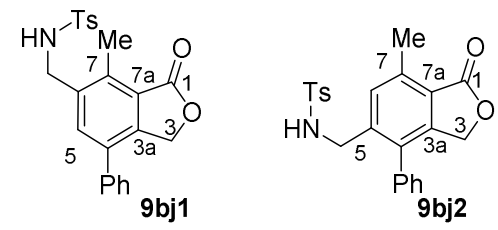

Obtained as a colorless liquid consisting of an inseparable mixture of $\mathbf{9 b j} \mathbf{1}$ and $\mathbf{9 b j} \mathbf{2}$ in an approx. ratio 1.5:1 (0.033 g, $0.082 \mathrm{mmol}, 63 \%$ yield). IR (film) $\left(\mathrm{cm}^{-1}\right): 3061,3026$, 2924, 1738, 1327, 1159, 1028. ${ }^{1} \mathrm{H}$ NMR $\left(\mathrm{CDCl}_{3}, 300 \mathrm{MHz}\right)$ : Major isomer 9bj1: $\delta$ 7.77$7.70(\mathrm{~m}, 2 \mathrm{H}, \mathrm{Ts}), 7.51(\mathrm{~s}, 1 \mathrm{H}, 5-\mathrm{H}), 7.49-7.36(\mathrm{~m}, 3 \mathrm{H}, \mathrm{Ar}-\mathrm{H}), 7.35-7.29(\mathrm{~m}, 2 \mathrm{H}$, Ar-H), 7.29-7.23 (m, 2H, Ts), $5.24(\mathrm{~s}, 2 \mathrm{H}, 3-\mathrm{H}), 5.04(\mathrm{t}, J=6.0 \mathrm{~Hz}, 1 \mathrm{H}, \mathrm{N}-\mathrm{H}), 4.26(\mathrm{~d}, J=6.0 \mathrm{~Hz}$, 2H, 6- $\left.\mathrm{CH}_{2}\right), 2.60\left(\mathrm{~s}, 3 \mathrm{H}, 7-\mathrm{CH}_{3}\right), 2.40(\mathrm{~s}, 3 \mathrm{H}, \mathrm{Ts})$. Minor isomer 9bj2: $\delta$ 7.59-7.53 (m, 2H, Ts), 7.49-7.36 (m, 2H, Ar-H), 7.29-7.23 (overlapping signal, 1H, Ar-H, 1H, 6-H), 7.23-7.16 (m, 2H, Ts), 7.14-7.07 (m, 2H, Ar-H), 4.90 (s, 2H, 3-H), 4.77 (t, J=6.2 Hz, 1H, N-H), 4.04 $\left(\mathrm{d}, J=6.3 \mathrm{~Hz}, 2 \mathrm{H}, 5-\mathrm{CH}_{2}\right), 2.61\left(\mathrm{~s}, 3 \mathrm{H}, 7-\mathrm{CH}_{3}\right), 2.40(\mathrm{~s}, 3 \mathrm{H}, \mathrm{Ts}) .{ }^{13} \mathrm{C} \mathrm{NMR}\left(\mathrm{CDCl}_{3}, 75\right.$ MHz): Major isomer 9bj1: $\delta 170.8$ (C, C-1), 144.1 (C, C-3a), 143.6 (C, Ts), 137.14 (C, C7), 137.08 (C, Ar), 136.53 (C, Ts), 136.50 (C, C-6), 134.1 (CH, C-5), 134.0 (C, C-4), 129.6 $(2 \times \mathrm{CH}, \mathrm{Ts}), 129.0(2 \times \mathrm{CH}, \mathrm{Ar}), 128.2(\mathrm{CH}, \mathrm{Ar}), 127.5(2 \times \mathrm{CH}, \mathrm{Ar}), 127.0(2 \times \mathrm{CH}, \mathrm{Ts})$, $124.1(\mathrm{C}, \mathrm{C}-7 \mathrm{a}), 68.1\left(\mathrm{CH}_{2}, \mathrm{C}-3\right), 44.2\left(\mathrm{CH}_{2}, \mathrm{C} 6-\mathrm{CH}_{2}\right), 21.4\left(\mathrm{CH}_{3}, \mathrm{Ts}\right), 12.2\left(\mathrm{CH}_{3}, \mathrm{C} 7-\right.$ $\mathrm{CH}_{3}$ ). Minor isomer 9bj2: $\delta 170.8(\mathrm{C}, \mathrm{C}-1), 146.5$ (C, C-3a), 143.5 (C, Ts), 139.8 (C, C-5), 138.8 (C, C-7), 136.5 (C, Ts), 134.8 (C, Ar), 133.4 (C, C-4), 131.6 (CH, C-6), 129.5 (2× 
$\mathrm{CH}, \mathrm{Ts}), 129.1(2 \times \mathrm{CH}, \mathrm{Ar}), 128.4(\mathrm{CH}, \mathrm{Ar}), 128.3(2 \times \mathrm{CH}, \mathrm{Ar}), 126.9(2 \times \mathrm{CH}, \mathrm{Ts})$, 122.7 (C, C-7a), $68.3\left(\mathrm{CH}_{2}, \mathrm{C}-3\right), 44.2\left(\mathrm{CH}_{2}, \mathrm{C} 5-\mathrm{CH}_{2}\right), 21.4\left(\mathrm{CH}_{3}, \mathrm{Ts}\right), 16.9\left(\mathrm{CH}_{3}, \mathrm{C} 7-\right.$ $\mathrm{CH}_{3}$ ). HRMS $m / z$ calcd. for $\mathrm{C}_{23} \mathrm{H}_{21} \mathrm{NNaO}_{4} \mathrm{~S}[\mathrm{M}+\mathrm{Na}]^{+} 430.1083$, found 430.1075.

\section{Compounds $4 \mathbf{j}$}

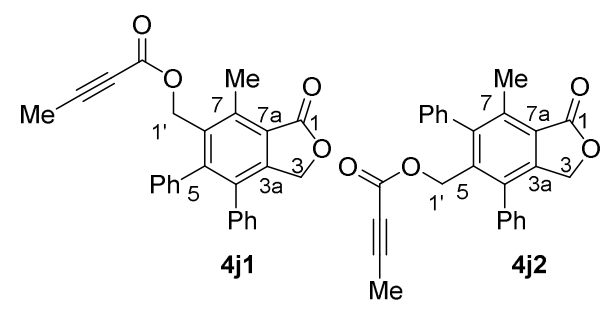

Obtained as a colorless liquid consisting of an inseparable mixture of $\mathbf{4} \mathbf{j} \mathbf{1}$ and $\mathbf{4} \mathbf{j} \mathbf{2}$ in an approx. ratio 2.4:1 (0.017 g, $0.043 \mathrm{mmol}, 13 \%$ yield). IR (film) $\left(\mathrm{cm}^{-1}\right): 3022,2961$, 2920, 2239, 1763, 1707, 1252. ${ }^{1} \mathrm{H}$ NMR $\left(\mathrm{CDCl}_{3}, 300 \mathrm{MHz}\right)$ : Major isomer 4j1: $\delta$ 7.52-6.92 (m, 10H, Ar-H), 5.05 (s, 2H, 1'-H), 5.04 (s, 2H, 3-H), 2.85 (s, 3H, 7-CH $), 1.98$ (s, 3H, 6’H). Minor isomer 4j2: $\delta$ 7.52-6.92 (m, 10H, Ar-H), $5.03(\mathrm{~s}, 2 \mathrm{H}, 3-\mathrm{H}), 4.67(\mathrm{~s}, 2 \mathrm{H}, 1$ '-H), $2.43\left(\mathrm{~s}, 3 \mathrm{H}, 7-\mathrm{CH}_{3}\right), 1.96\left(\mathrm{~s}, 3 \mathrm{H}, 6{ }^{\prime}-\mathrm{H}\right) .{ }^{13} \mathrm{C} \mathrm{NMR}\left(\mathrm{CDCl}_{3}, 75 \mathrm{MHz}\right)$ : Major isomer 4j1: $\delta$ 170.9 (C, C-1), 153.0 (C, C-3'), 148.2 (C, C-5), 146.5 (C, C-3a), 140.0 (C, C-6), 134.3 (C, C-7), 122.8 (C, C-7a), 86.1 (C, C-5'), 71.8 (C, C-4'), $68.2\left(\mathrm{CH}_{2}, \mathrm{C}-3\right), 62.4\left(\mathrm{CH}_{2}, \mathrm{C}^{\prime} 1^{\prime}\right)$, $13.1\left(\mathrm{CH}_{3}, 7-\mathrm{CH}_{3}\right), 3.7\left(\mathrm{CH}_{3}, \mathrm{C}-6\right.$ '). Minor isomer 4j2: $\delta 171.0(\mathrm{C}, \mathrm{C}-1), 152.3\left(\mathrm{C}, \mathrm{C}-3^{\prime}\right)$, 145.8 (C, C-6), 145.3 (C, C-3a), 137.8 (C, C-7), 136.1 (C, C-5), 123.6 (C, C-7a), 85.6 (C, C-5'), 71.8 (C, C-4'), $68.2\left(\mathrm{CH}_{2}, \mathrm{C}-3\right), 62.5\left(\mathrm{CH}_{2}, \mathrm{C}-1^{\prime}\right), 14.9\left(\mathrm{CH}_{3}, 7-\mathrm{CH}_{3}\right), 3.7\left(\mathrm{CH}_{3}, \mathrm{C}-\right.$ 6'). Unassigned signals: $\delta 137.4,137.3,136.3,135.8,135.4,133.3,129.7,129.1,128.93$, $128.88,128.6,128.5,128.4,128.2,127.8,127.7,127.5,127.3$. HRMS $m / z$ calcd. for $\mathrm{C}_{26} \mathrm{H}_{21} \mathrm{O}_{4}[\mathrm{M}+\mathrm{H}]^{+}$397.1434, found 397.1428. 


\section{Compounds 4'j}

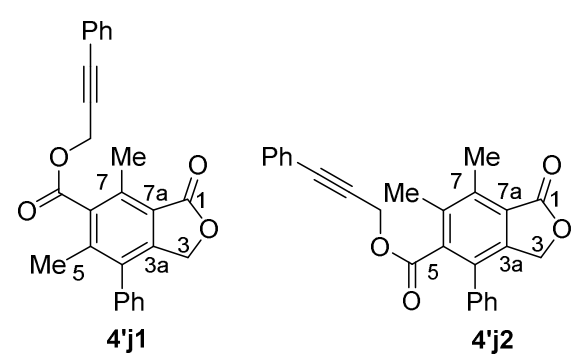

Obtained as a pale yellow liquid consisting of an inseparable mixture of 4'j1 and 4'j2 in an approx. ratio 1.2:1 (0.020 g, $0.049 \mathrm{mmol}, 15 \%$ yield). IR (film) $\left(\mathrm{cm}^{-1}\right): 3057$, 3022, 2926, 2228, 1759, 1736, 1443, 1300, 1207, 1159, 1026. ${ }^{1} \mathrm{H}$ NMR $\left(\mathrm{CDCl}_{3}, 300 \mathrm{MHz}\right)$ : Major isomer 4'j1: $\delta$ 7.52-7.15 (m, 10H, Ar-H), 5.21 (s, 2H, 3'-H), 4.95 (s, 2H, 3-H), 2.74 (s, 3H, 7-CH 3$), 2.23\left(\mathrm{~s}, 3 \mathrm{H}, 5-\mathrm{CH}_{3}\right)$. Minor isomer 4'j2: $\delta$ 7.52-7.15 (m, 10H, Ar-H), 5.04 $(\mathrm{s}, 2 \mathrm{H}, 3-\mathrm{H}), 4.84\left(\mathrm{~s}, 2 \mathrm{H}, 3\right.$ '-H), $2.72\left(\mathrm{~s}, 3 \mathrm{H}, 7-\mathrm{CH}_{3}\right), 2.40\left(\mathrm{~s}, 3 \mathrm{H}, 6-\mathrm{CH}_{3}\right) .{ }^{13} \mathrm{C} \mathrm{NMR}$ $\left(\mathrm{CDCl}_{3}, 75 \mathrm{MHz}\right)$ : Major isomer 4’j1: $\delta 170.7$ (C, C-1), 168.3 (C, C-1'), 147.1 (C, C-3a), 139.1 (C, C-5), 136.2 (C, C-6), 135.3 (C, C-7), 134.7 (C, C-4), 131.7 (2 × CH, C-7’), 121.7 (C, C-6’), 121.1 (C, C-7a), 87.2 (C, C-5'), 82.1 (C, C-4'), $68.2\left(\mathrm{CH}_{2}, \mathrm{C}-3\right), 53.7\left(\mathrm{CH}_{2}, \mathrm{C}-\right.$ 3'), $17.5\left(\mathrm{CH}_{3}, \mathrm{C} 5-\mathrm{CH}_{3}\right), 14.0\left(\mathrm{CH}_{3}, \mathrm{C} 7-\mathrm{CH}_{3}\right)$. Minor isomer 4'j2: $\delta 170.9(\mathrm{C}, \mathrm{C}-1), 167.9$ (C, C-1'), 143.2 (C, C-3a), 138.2 (C, C-6), 138.1 (C, C-7), 135.8 (C, C-4), 135.5 (C, C-5), 131.8 (2 × CH, C-7’'), 123.8 (C, C-7a), 121.9 (C, C-6’'), 86.8 (C, C-5'), 81.8 (C, C-4'), 67.8 $\left(\mathrm{CH}_{2}, \mathrm{C}-3\right), 53.4\left(\mathrm{CH}_{2}, \mathrm{C}-3\right.$ ') $, 16.2\left(\mathrm{CH}_{3}, \mathrm{C} 6-\mathrm{CH}_{3}\right), 13.1\left(\mathrm{CH}_{3}, \mathrm{C} 7-\mathrm{CH}_{3}\right)$. Unassigned signals: $\delta 131.6,129.0,128.83,128.77,128.74,128.4,128.3,128.24,128.19$. HRMS m/z calcd. for $\mathrm{C}_{26} \mathrm{H}_{20} \mathrm{NaO}_{4}[\mathrm{M}+\mathrm{Na}]^{+} 419.1254$, found 419.1247 . 


\section{Compounds $10 \mathrm{c1} / \mathrm{c2}^{33}$}
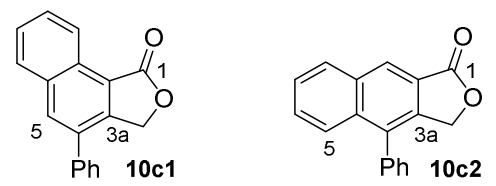

Obtained as a pale yellow solid consisting of an inseparable mixture of 10c1 and 10c2 in an approx. ratio 1.25:1 (0.013 g, $0.05 \mathrm{mmol}, 8 \%$ yield). M.p.: $101.8-108.3{ }^{\circ} \mathrm{C}$. IR (film) $\left(\mathrm{cm}^{-1}\right): 3057,3028,2930,1755,1026 .{ }^{1} \mathrm{H} \mathrm{NMR}\left(\mathrm{CDCl}_{3}, 300 \mathrm{MHz}\right)$ : Major isomer 10c1: $\delta 9.05(\mathrm{~d}, J=8.3 \mathrm{~Hz}, 1 \mathrm{H}, 9-\mathrm{H}), 8.12(\mathrm{~s}, 1 \mathrm{H}, 5-\mathrm{H}), 8.00(\mathrm{~d}, J=8.1 \mathrm{~Hz}, 1 \mathrm{H}, 6-\mathrm{H}), 7.72$ $(\mathrm{ddd}, J=8.2 \mathrm{~Hz}, J=7.0 \mathrm{~Hz}, J=1.3 \mathrm{~Hz}, 1 \mathrm{H}, 8-\mathrm{H}), 7.65(\mathrm{ddd}, J=8.2 \mathrm{~Hz}, J=7.2 \mathrm{~Hz}, J=$ $1.3 \mathrm{~Hz}, 1 \mathrm{H}, 7-\mathrm{H}), 7.61-7.36(\mathrm{~m}, 5 \mathrm{H}, \mathrm{Ar}-\mathrm{H}), 5.43(\mathrm{~s}, 2 \mathrm{H}, 3-\mathrm{H})$. Minor isomer 10c2: $\delta 8.51$ (s, 1H, 9-H), 8.11-8.05 (m, 1H, Ar-H), 7.84-7.78 (m, 1H, 8-H), 7.61-7.36 (m, 7H, Ar-H), $5.26(\mathrm{~s}, 2 \mathrm{H}, 3-\mathrm{H}) .{ }^{13} \mathrm{C} \mathrm{NMR}\left(\mathrm{CDCl}_{3}, 75 \mathrm{MHz}\right)$ : Major isomer $10 \mathrm{c1}$ (C-9a signal missing): $\delta$ 171.3 (C, C-1), 146.9 (C, C-3a), 137.6 (C, Ph), 134.0 (CH, C-5), 133.8 (C, C-4), 133.7 (C, C-5a), $128.9(\mathrm{CH}, \mathrm{C}-8), 128.4(3 \times \mathrm{CH}, \mathrm{C}-6, \mathrm{Ph}), 128.3(2 \times \mathrm{CH}, \mathrm{Ph}), 127.5(\mathrm{CH}, \mathrm{C}-7)$, $123.2(\mathrm{CH}, \mathrm{C}-9), 120.5$ (C, C-9b), $69.1\left(\mathrm{CH}_{2}, \mathrm{C}-3\right)$. Minor isomer 10c2: $\delta 171.1(\mathrm{C}, \mathrm{C}-1)$, 138.3 (C, C-3a), 134.7 (C, C-4a), 126.3 (CH, C-9), 125.8 (CH, C-8), 122.9 (C, C-9a), 69.4 $\left(\mathrm{CH}_{2}, \mathrm{C}-3\right)$. Unassigned signals: $\delta 135.7,133.6,130.0,129.2,129.1,128.9,128.7,127.8$, 126.6 .

\section{Compounds 10c3/c4 $4^{34}$}

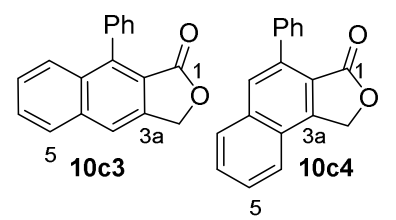


Obtained as a pale yellow solid consisting of an inseparable mixture of 10c3 and 10c4 in an approx. ratio 1.2:1 (0.013 g, $0.05 \mathrm{mmol}, 8 \%$ yield). M.p.: $130.4-137.7{ }^{\circ} \mathrm{C}$. IR (film) $\left(\mathrm{cm}^{-1}\right): 3057,3022,2928,1763,1045,1028 .{ }^{1} \mathrm{H} \mathrm{NMR}\left(\mathrm{CDCl}_{3}, 300 \mathrm{MHz}\right): 10 \mathrm{c3}: \delta$ $7.96(\mathrm{~d}, J=8.2 \mathrm{~Hz}, 1 \mathrm{H}, 5-\mathrm{H}), 7.90$ (s, 1H, 4-H), 7.81 (d, $J=8.6 \mathrm{~Hz}, 1 \mathrm{H}, 8-\mathrm{H}), 7.69-7.34$ (m, 7H, Ar-H, 6-H, 7-H), 5.44 (bs, 2H, 3-H). 10c4: $\delta 8.01$ (d, $J=8.0 \mathrm{~Hz}, 1 \mathrm{H}, 7-\mathrm{H}), 7.86$ (s, 1H, 8-H), 7.85 (d, $J=7.6 \mathrm{~Hz}, 1 \mathrm{H}, 4-\mathrm{H}), 7.72$ (td, $J=6.8 \mathrm{~Hz}, J=1.0 \mathrm{~Hz}, 1 \mathrm{H}, 6-\mathrm{H}), 7.69-$ $7.34(\mathrm{~m}, 6 \mathrm{H}, \mathrm{Ar}-\mathrm{H}, 5-\mathrm{H}), 5.64(\mathrm{~s}, 2 \mathrm{H}, 3-\mathrm{H}) .{ }^{13} \mathrm{C} \mathrm{NMR}\left(\mathrm{CDCl}_{3}, 75 \mathrm{MHz}\right): 10 \mathrm{c3}: \delta 169.5(\mathrm{C}$, C-1), 142.1 (C, C-9), 140.0 (C, C-3a), 136.1 (C, C-4a), 132.7 (C, C-8a), 126.6 (CH, C-7), $120.2(\mathrm{CH}, \mathrm{C}-4), 68.0\left(\mathrm{CH}_{2}, \mathrm{C}-3\right) .10 \mathrm{c} 4: \delta 170.2$ (C, C-1), 148.4 (C, C-3a), 136.5 (C, C-9), 135.6 (C, C-7a), 130.5 (CH, C-8), 129.6 (CH, C-6), 128.9 (CH, C-7), 127.5 (CH, C-5), 125.9 (C, C-3b), 123.0 (CH, C-4), 120.1 (C, C-9a), $67.7\left(\mathrm{CH}_{2}, \mathrm{C}-3\right)$. Unassigned signals: $\delta$ $137.3,134.3,129.9,128.5,128.2,128.0,127.9,127.8,127.5,119.8$.

\section{Compound 10h1 $1^{35}$}

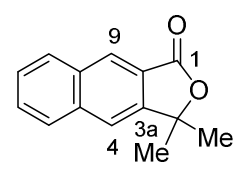

Obtained as a yellow liquid consisting of a difficult to separate mixture of $\mathbf{1 0 h} \mathbf{1}$ and $\mathbf{1 0 h 2}$ in a ratio 1.5:1 (0.008 g, $0.04 \mathrm{mmol}, 6 \%$ yield $).{ }^{1} \mathrm{H}$ NMR $\left(\mathrm{CDCl}_{3}, 300 \mathrm{MHz}\right): \delta 8.44(\mathrm{~s}$, 1H), $8.02(\mathrm{~d}, J=8.1 \mathrm{~Hz}, 1 \mathrm{H}), 7.94(\mathrm{~d}, J=8.2 \mathrm{~Hz}, 1 \mathrm{H}), 7.81$ (s, 1H), 7.68-7.59 (overlapping signal, 1H), 7.57 (ddd, $J=8.2 \mathrm{~Hz}, J=7.0 \mathrm{~Hz}, J=1.0 \mathrm{~Hz}, 1 \mathrm{H}), 1.76(\mathrm{~s}, 6 \mathrm{H})$. 


\section{Compound 10h2}

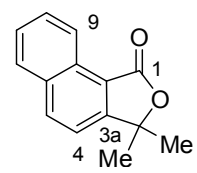

Obtained as a pale yellow liquid $(0.013 \mathrm{~g}, 0.06 \mathrm{mmol}, 9 \%$ yield $)$. IR (film) $\left(\mathrm{cm}^{-1}\right)$ : 3057, 2978, 2928, 1747, 1518, 1306, 1082. ${ }^{1} \mathrm{H}$ NMR $\left(\mathrm{CDCl}_{3}, 300 \mathrm{MHz}\right): \delta 9.02(\mathrm{~d}, J=8.4$ Hz, 1H, 9-H), 8.14 (d, $J=8.4 \mathrm{~Hz}, 1 \mathrm{H}, 5-\mathrm{H}), 7.96$ (d, $J=8.4 \mathrm{~Hz}, 1 \mathrm{H}, 6-\mathrm{H}), 7.73$ (ddd, $J=$ $8.2 \mathrm{~Hz}, J=7.0 \mathrm{~Hz}, J=1.1 \mathrm{~Hz}, 1 \mathrm{H}, 8-\mathrm{H}), 7.62(\mathrm{ddd}, J=8.1 \mathrm{~Hz}, J=7.0 \mathrm{~Hz}, J=1.1 \mathrm{~Hz}, 1 \mathrm{H}$, 7-H), $7.45(\mathrm{~d}, J=8.5 \mathrm{~Hz}, 1 \mathrm{H}, 4-\mathrm{H}), 1.72\left(\mathrm{~s}, 6 \mathrm{H}, 3-\mathrm{CH}_{3}\right) .{ }^{13} \mathrm{C} \mathrm{NMR}\left(\mathrm{CDCl}_{3}, 75 \mathrm{MHz}\right): \delta$ 170.0 (C, C-1), 156.4 (C, C-3a), 135.5 (CH, C-5), 133.1 (C, C-5a), 129.2 (C, C-9a), 129.0 (CH, C-8), 128.3 (CH, C-6), 127.0 (CH, C-7), 123.6 (CH, C-9), 119.0 (C, C-9b), 117.5 $(\mathrm{CH}, \mathrm{C}-4), 84.1(\mathrm{C}, \mathrm{C}-3), 26.9\left(2 \times \mathrm{CH}_{3}, \mathrm{C} 3-\mathrm{CH}_{3}\right)$. HRMS $m / z$ calcd. for $\mathrm{C}_{14} \mathrm{H}_{13} \mathrm{O}_{2}[\mathrm{M}+$ $\mathrm{H}]^{+}$213.0910, found 213.0916.

\section{AUTHOR CONTRIBUTIONS}

The manuscript was written through contributions of all authors. / All authors have given approval to the final version of the manuscript. / ${ }^{\dagger}$ M.J.R. and C.M.D. contributed equally to this work.

\section{NOTES}

The authors declare no competing financial interest.

\section{SUPPORTING INFORMATION}

The Supporting Information is available free of charge on the ACS Publications website. Copies of ${ }^{1} \mathrm{H},{ }^{13} \mathrm{C}$ and 2D NMR spectra for all new compounds (PDF). 


\section{ACKNOWLEDGEMENTS}

Support from CONICET (PUE-2016), ANPCyT (PICT.2014-0408), Agencia Santafesina de Ciencia, Técnica e Innovación (ASACTEI) (AC - 2015-00005) and Universidad Nacional de Rosario (BIO 426, BIO 514). C.M.D. thanks CONICET for fellowships.

\section{REFERENCES AND FOOTNOTES}

(1) Transition-metal-mediated Aromatic Ring Construction, Tanaka, K., Ed.; John Wiley \& Sons Hoboken, 2013; Part I, 3-320.

(2) For example, see: Nicolaou, K. C.; Wang, J.; Tang, Y.; Botta, L.; Total Synthesis of Sporolide B and 9-epi-Sporolide B. J. Am. Chem. Soc. 2010, 132, 11350-11363.

(3) Pla-Quintana, A.; Roglans, A.; [2+2+2] Cycloaddition Reactions of Macrocyclic Systems Catalyzed by Transition Metals. A Review. Molecules 2010, 15, 9230-9251.

(4) For example, see: (a) Lynett, P. T.; Maly, K. E.; Synthesis of Substituted Trinaphthylenes via Aryne Cyclotrimerization. Org. Lett. 2009, 11, 3726-3729. (b) Shen, X.; Ho, D. M.; Pascal, R. A. Jr.; Synthesis of Polyphenylene Dendrimers Related to "Cubic Graphite”. J. Am. Chem. Soc. 2004, 126, 5798-5805.

(5) For example, see: Sakiyama, N.; Hojo, D.; Noguchi, K.; Tanaka, K.; Enantioselective Synthesis of Axially Chiral 1-Arylisoquinolines by Rhodium-Catalyzed [2+2+2] Cycloaddition. Chem. Eur. J. 2011, 17, 1428-1432.

(6) For reviews, see: (a) Domínguez, G.; Pérez-Castells, J.; [2+2+2] Cycloadditions. In Comprehensive Organic Synthesis II, Knochel, P., Molaner, G., Eds ; Elsevier Amsterdam, 2014, 5, 1537-1581. (b) Domínguez, G.; Pérez-Castells, J.; Recent Advances in [2+2+2] Cycloaddition Reactions. Chem. Soc. Rev. 2011, 40. 3430-3444. (c) Shaaban, M. R.; ElSayed, R.; Elwahy, A. H. M.; Construction of Fused Heterocycles by Metal-mediated 
[2+2+2] Cyclotrimerization of Alkynes and/or Nitriles. Tetrahedron 2011, 67, 6095-6130.

(d) Shibata, Y.; Tanaka, K.; Rhodium-Catalyzed [2+2+2] Cycloaddition of Alkynes for the Synthesis of Substituted Benzenes: Catalysts, Reaction Scope, and Synthetic Applications. Synthesis 2012, 44, 323-350. (e) Galan, B. R.; Rovis, T.; Beyond Reppe: Building Substituted Arenes by [2+2+2] Cycloadditions of Alkynes. Angew. Chem. Int. Ed. 2009, 48, 2830-2834. (f) Shibata, T.; Tsuchikama, K.; Recent Advances in Enantioselective [2+2+2] Cycloaddition. Org. Biomol. Chem. 2008, 6, 1317-1323. (g) Chopade, P. R.; Louie, J.; [2+2+2] Cycloaddition Reactions Catalyzed by Transition Metal Complexes. Adv. Synth. Catal. 2006, 348. 2307-2327. (h) Gandon, V.; Aubert, C.; Malacria, M.; Recent Progress in Cobalt-mediated [2+2+2] Cycloaddition Reactions. Chem. Commun. 2006, 2209-2217. (i) Kotha, S.; Brahmachary, E.; Lahiri, K.; Transition Metal Catalyzed [2+2+2] Cycloaddition and Application in Organic Synthesis. Eur. J. Org. Chem. 2005, 4741-4767.

(7) For example, see: (a) Xu, H.-D.; Zhang, R.-W.; Li, X.; Huang, S.; Tang, W.; Hu, W.-H.; Rhodium-Catalyzed Chemo- and Regioselective Cross-Dimerization of Two Terminal Alkynes. Org Lett. 2013, 15, 840-843. (b) Hoshino, Y.; Shibata, Y.; Tanaka, K.; RhodiumCatalyzed Three-Component Cross-Addition of Silylacetylenes, Alkynyl Esters, and Electron-Deficient Alkenes or Isocyanates. Angew. Chem. Int. Ed. 2012, 51, 9407-9411. (c) Oh, C. H.; Sung, H. R.; Jung, S. H.; Lim, Y. M.; Rhodium Catalyzed [2+2+2] Cyclizations of 1,6-Enynes. Tetrahedron Lett. 2001, 42, 5493-5495.

(8) Takeuchi, R.; Nakaya, Y.; Iridium Complex-Catalyzed Highly Selective Cross [2+2+2] Cycloaddition of Two Different Monoynes: 2:1 Coupling versus 1:2 Coupling. Org. Lett. 2003, 5, 3659-3662.

(9) Yamamoto, Y.; Ishii, J.; Nishiyama, H.; Itoh, K.; Cp*RuCl-Catalyzed Formal Intermolecular Cyclotrimerization of Three Unsymmetrical Alkynes through a Boron 
Temporary Tether: Regioselective Four-Component Coupling Synthesis of Phthalides. J. Am. Chem. Soc. 2005, 127, 9625-9631.

(10) (a) Chouraqui, G.; Petit, M.; Aubert, C.; Malacria, M.; Totally Chemo- and Regioselective Cobalt(I)-Mediated Formal Intermolecular Cyclotrimerization of Alkynes. Org. Lett. 2004, 6, 1519-1521. (b) Chouraqui, G.; Petit, M.; Phansavath, P.; Aubert, C.; Malacria, M.; From an acyclic, polyunsaturated precursor to the polycyclic taxane ring system: the $[4+2] /[2+2+2]$ and $[2+2+2] /[4+2]$ cyclization strategies. Eur. J. Org. Chem. 2006, 1413-1421.

(11) For example, see: Riveira, M. J.; Mata, E. G.; Cross-Metathesis on Immobilized Substrates - Application to the Generation of Synthetically and Biologically Relevant Structures. Eur. J. Org. Chem. 2017, 1675-1693.

(12) Sun, Q.; Zhou, X.; Islam, K.; Kyle, D. J.; Solid-phase Synthesis of Isoindolines via a Rhodium-catalyzed [2+2+2] Cycloaddition. Tetrahedron Lett. 2001, 42, 6495-6497.

(13) (a) Senaiar, R. S.; Teske, J. A.; Young, D. D.; Deiters, A.; Synthesis of Indanones via Solid-Supported [2+2+2] Cyclotrimerization. J. Org. Chem. 2007, 72, 7801-7804. (b) Young, D. D.; Sripada, L.; Deiters, A.; Microwave-Assisted Solid-Supported Alkyne Cyclotrimerization Reactions for Combinatorial Chemistry. J. Comb. Chem. 2007, 9, 735738. (c) Young, D. D.; Senaiar, R. S.; Deiters, A.; Solid-Supported [2+2+2] Cyclotrimerizations. Chem. Eur. J. 2006, 12, 5563-5568.

(14) Shanmugasundaram, M.; Aguirre, A. L.; Leyva, M.; Quan, B.; Martinez, L. E.; Microwave-assisted Iridium-catalyzed [2+2+2] Cycloaddition of Resin-bound Dipropargylamine with Alkynes. Tetrahedron Lett. 2007, 48, 7698-7701.

(15) Witulski, B.; Zimmermann, A.; Synthesis of Chiral 3-Substituted Phthalides via Rhodium(I)-catalyzed Crossed Alkyne Cyclotrimerisation. Synlett 2002, 1855-1859. 
(16) For a recent review on phthalides and their preparation, see: Karmakar, R.; Pahari, P.; Mal, D.; Phthalides and Phthalans: Synthetic Methodologies and Their Applications in the Total Synthesis. Chem. Rev. 2014, 114, 6213-6284.

(17) Lin, G.; Chan, S. S.-K.; Chung, H.-S.; Li, S.-L.; Chemistry and Biological Activities of Naturally Occurring Phthalides. In "Studies in Natural Products Chemistry"; Rahman, A., Ed.; Elsevier, Amsterdam, 2005, Vol. 32, pp 611-671.

(18) For previous examples on the preparation of phthalides via $[2+2+2]$-cycloadditions, see: (a) Tanaka, K.; Osaka, T.; Noguchi, K.; Hirano, M.; Rhodium-Catalyzed Asymmetric One-Pot Transesterification and [2+2+2] Cycloaddition Leading to Enantioenriched 3,3Disubstituted Phthalides. Org. Lett. 2007, 9, 1307-1310. (b) Chang, H.-T.; Jeganmohan, M.; Cheng, C.-H.; One-pot Synthesis of Benzolactones and Lactams via a Cobalt-catalyzed Regioselective [2+2+2] Cocyclotrimerization of Alkynyl Alcohols and Amines with Propiolates. Chem. Commun. 2005, 4955-4957. (c) Yamamoto, Y.; Kinpara, K.; Saigoku, T.; Nishiyama, H.; Itoh K.; Synthesis of Benzo-fused Lactams and Lactones via Ru(II)Catalyzed Cycloaddition of Amide- and Ester-tethered $\alpha, \omega$-Diynes with Terminal Alkynes: Electronic Directing Effect of Internal Conjugated Carbonyl Group. Org. Biomol. Chem. 2004, 2, 1287-1294. (d) Tanaka, K.; Nishida, G.; Wada, A.; Noguchi, K.; Enantioselective Synthesis of Axially Chiral Phthalides through Cationic [ $\mathrm{Rh}^{\mathrm{I}}\left(\mathrm{H}_{8}\right.$-binap)]-Catalyzed Cross Alkyne Cyclotrimerization. Angew. Chem. Int. Ed. 2004, 43, 6510-6512. (e) Sato, Y.; Ohashi, K.; Mori, M.; Synthesis of Biaryls Using Nickel-Catalyzed [2+2+2] Cocyclization. Tetrahedron Lett. 1999, 40, 5231-5234. (f) Neeson, S. J.; Stevenson, P. J.; Rhodium Catalysed [2+2+2] Cycloadditions-An Efficient Regiospecific Route to Calomelanolactone. Tetrahedron Lett. 1988, 29, 813-814. For some syntheses of natural phthalides via [2+2+2]- 
cycloadditions, see: (g) Zou, Y.; Deiters, A.; Total Synthesis of Cryptoacetalide. J. Org. Chem. 2010, 75, 5355-5358. (h) Welsch, T.; Tran, H.-A.; Witulski, B.; Total Syntheses of the Marine Illudalanes Alcyopterosin I, L, M, N, and C. Org. Lett. 2010, 12, 5644-5647. (i) Sato, Y.; Tamura, T.; Mori, M.; Arylnaphthalene Lignans through Pd $\square$ Catalyzed [2+2+2] Cocyclization of Arynes and Diynes: Total Synthesis of Taiwanins C and E. Angew. Chem. Int. Ed. 2004, 43, 2436-2440. (j) Witulski, B.; Zimmermann, A.; Gowans, N. D. First Total Synthesis of the Marine Illudalane Sesquiterpenoid Alcyopterosin E. Chem. Commun. 2002, 0, 2984-2985.

(19) White, P. D. in "The Power of Functional Resins in Organic Synthesis"; Tulla-Puche, J.; Albericio, F., Eds.; Wiley-VCH, Weinheim, 2008, pp 383-413.

(20) (a) René, O.; Fauber, B. P.; de Leon Boenig, G.; Burton, B.; Eidenschenk, C.; Everett, C.; Gobbi, A.; Hymowitz, S. G.; Johnson, A. R.; Kiefer, J. R.; Liimatta, M.; Lockey, P.; Norman, M.; Ouyang, W.; Wallweber, H. A.; Wong, H.; Minor Structural Change to Tertiary Sulfonamide RORc Ligands Led to Opposite Mechanisms of Action. ACS Med. Chem. Lett. 2015, 6, 276-281. (b) Hennessy, E. J.; Oza, V.; Adam, A.; Byth, K.; Castriotta, L.; Grewal, G.; Hamilton, G. A.; Kamhi, V. M.; Lewis, P.; Li, D.; Lyne, P.; Öster, L.; Rooney, M. T.; Saeh, J. C.; Sha, L.; Su, Q.; Wen, S.; Xue, Y.; Yang, B.; Identification and Optimization of Benzimidazole Sulfonamides as Orally Bioavailable Sphingosine 1Phosphate Receptor 1 Antagonists with in Vivo Activity. J. Med. Chem. 2015, 58, 70577075. (c) Rettenmaier, T. J.; Sadowsky, J. D.; Thomsen, N. D.; Chen, S. C.; Doak, A. K.; Arkin, M. R.; Wells, J. A.; A Small-molecule Mimic of a Peptide Docking Motif Inhibits the Protein Kinase PDK1. Proc. Natl. Acad. Sci. USA 2014, 111, 18590-18595. (d) Shao, P. P.; Ye, F.; Chakravarty, P. K.; Varughese, D. J.; Herrington, J. B.; Dai, G.; Bugianesi, R. M.; Haedo, R. J.; Swensen, A. M.; Warren, V. A.; Smith, M. M.; Garcia, M. L.; McManus, O. 
B.; Lyons, K. A.; Li, X.; Green, M.; Jochnowitz, N.; McGowan, E.; Mistry, S.; Sun, S.-Y.; Abbadie, C.; Kaczorowskiand, G. J.; Duffy, J. L.; Aminopiperidine Sulfonamide Ca 2.2 Channel Inhibitors for the Treatment of Chronic Pain. J. Med. Chem. 2012, 55, 9847-9855. (e) Zuercher, W. J.; Buckholz, R. G.; Campobasso, N.; Collins, J. L.; Galardi, C. M.; Gampe, R. T.; Hyatt, S. M.; Merrihew, S. L.; Moore, J. T.; Oplinger, J. A.; Reid, P. R.; Spearing, P. K.; Stanley, T. B.; Stewart, E. L.; Willson, T. M.; Discovery of Tertiary Sulfonamides as Potent Liver X Receptor Antagonists. J. Med. Chem. 2010, 53, 3412-3416. (f) Barton, N. P.; Bellenie, B. R.; Doran, A. T.; Emmons, A. J.; Heer, J. P.; Salvagno, C. M.; Discovery and Optimisation of a Potent and Selective Tertiary Sulfonamide Oxytocin Antagonist. Bioorg. Med. Chem. Lett. 2009, 19, 528-532.

(21) Yamamoto, Y.; Kuwabara, S.; Ando, Y.; Nagata, H.; Nishiyama, H.; Itoh, K.; Palladium(0)-Catalyzed Cyclization of Electron-Deficient Enynes and Enediynes. J. Org. Chem. 2004, 69, 6697-6705.

(22) Yuan, W.; Wei, Y.; Shi, M.; Ruthenium-Catalyzed Intramolecular [2+2+2] Cycloaddition and Tandem Cross-Metathesis of Triynes and Enediynes. ChemistryOpen 2013, 2, 63-68.

(23) Pure samples of some homodimers and homotrimers 5d were obtained by performing the corresponding cycloadditions in the absence of the monoyne component 1a. See ESI. (24) Kobayashi, S.; Aoki, Y.; p-Benzyloxybenzylamine (BOBA) Resin. A New PolymerSupported Amine Used in Solid-Phase Organic Synthesis. Tetrahedron Lett. 1998, 39, $7345-7348$.

(25) For examples on the use of BOBA or related resins, see: (a) Larsen, S. D.; DiPaolo, B. A.; Traceless Solid-Phase Synthesis of 1,2,4-Triazoles Using a Novel Amine Resin. Org. Lett. 2001, 3, 3341-3344. (b) Aoki, Y.; Kobayashi, S.; Development of a Reductive 
Alkylation Method Using $p$-Benzyloxybenzylamine (BOBA) Resin for the Synthesis of NAlkylated Amides. J. Comb. Chem. 1999, 1, 371-372.

(26) It is noteworthy that this side reaction was not observed in solution experiments and, in this regard, we assume this is due to a lower reaction rate on solid-phase as it was found that, for example, the [2+2+2]-cycloaddition between 1a and $\mathbf{2 a}$ was complete in $30 \mathrm{~min}$.

(27) For two reviews on DDA reactions, see: (a) Li, W.; Zhou, L.; Zhang, J.; Recent Progress in Dehydro(genative) Diels-Alder Reaction. Chem. Eur. J. 2016, 22, 1558-1571.

(b) Wessig, P.; Müller, G.; The Dehydro-Diels-Alder Reaction. Chem. Rev. 2008, 108, 2051-2063.

(28) Kirchner, K.; Calhorda, M. J.; Schmid, R.; Veiros, L. F.; Mechanism for the Cyclotrimerization of Alkynes and Related Reactions Catalyzed by CpRuCl. J. Am. Chem. Soc. 2003, 125, 11721-11729.

(29) (a) Amatore, M.; Lebœuf, D.; Malacria, M.; Gandon, V.; Aubert, C.; Highly Enantioselective Rhodium-Catalyzed [2+2+2] Cycloaddition of Diynes to Sulfonimines. $J$. Am. Chem. Soc. 2013, 135, 4576-4579. (b) Barbazanges, M.; Caytan, E.; Lesage, D.; Aubert, C.; Fensterbank, L.; Gandon, V.; Ollivier, C.; Chiral Phosphate in Rhodium-Catalyzed Asymmetric [2+2+2] Cycloaddition: Ligand, Counterion, or Both?. Chem. Eur. J. 2016, 22, 8553-8558.

(30) Huang, W.; Shen, Q.: Wang, J.; Zhou, X.; One-step Synthesis of Substituted Dihydroand Tetrahydroisoquinolines by $\mathrm{FeCl}_{3} \cdot 6 \mathrm{H}_{2} \mathrm{O}$ Catalyzed Intramolecular Friedel-Crafts Reaction of Benzylamino-Substituted Propargylic Alcohols. J. Org. Chem. 2008, 73, 15861589.

(31) (a) Onodera, G.; Suto, M.; Takeuchi, R.; Iridium-Catalyzed [2+2+2] Cycloaddition of

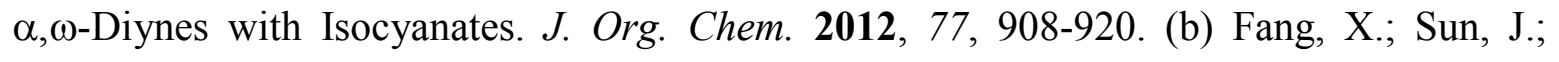


Tong, X.; Rh(I)-catalyzed Formal [2+2+2] Cycloadditions of 1,6-Diynes with Potassium (Z)-(2-Bromovinyl)trifluoroborate: A New Strategy and a Facile Entry to Polysubstituented Benzene Derivatives. Chem. Commun. 2010, 46, 3800-3802. (c) Klemm, L. H.; Klemm, R. A.; Santhanam, P. S.; White, D. V.; Intramolecular Diels-Alder Reactions. VI. Synthesis of 3-Hydroxymethyl-2-naphthoic Acid Lactones. J. Org. Chem. 1971, 36, 2169-2172.

(32) Liu, Y.; Huang, Y.; Song, H.; Liu, Y.; Wang, Q.; Regio- and Chemoselective N-1 Acylation of Indoles: Pd-Catalyzed Domino Cyclization to Afford 1,2-Fused Tricyclic Indole Scaffolds. Chem. Eur. J. 2015, 21, 5337-5340.

(33) Kao, T.-T.; Lin, C.-C.; Shia, K.-S.; The Total Synthesis of Retrojusticidin B, Justicidin E, and Helioxanthin. J. Org. Chem. 2015, 80, 6708-6714.

(34) Anderson, M. R.; Brown, R. F. C.; Coulston, K. J.; Eastwood, F. W.; Ward, A.; The Pyrolysis of Phenylnaphthalenedicarboxylic Anhydrides: Products of Ring Contraction and of Radical Cyclization. Aust. J. Chem. 1990, 43, 1137-1150.

(35) Nishio, T.; Photoreactions of 1,3-Dihydroisobenzofuran-1-thiones and 1,3-Dihydro-2benzothiophene-1-thiones with Alkenes. J. Chem. Soc., Perkin Trans. 1 (1972-1999) 1995, $561-568$. 